\title{
A novel CMB polarization likelihood package for large angular scales built from combined WMAP and Planck LFI legacy maps
}

\author{
U. Natale ${ }^{1,2}$, L. Pagano ${ }^{1,2}$, M. Lattanzi ${ }^{2}$, M. Migliaccio ${ }^{3,4}$, L. P. Colombo ${ }^{5}$, A. Gruppuso ${ }^{6,7}$, \\ P. Natoli ${ }^{1,2}$, and G. Polenta ${ }^{8}$
}

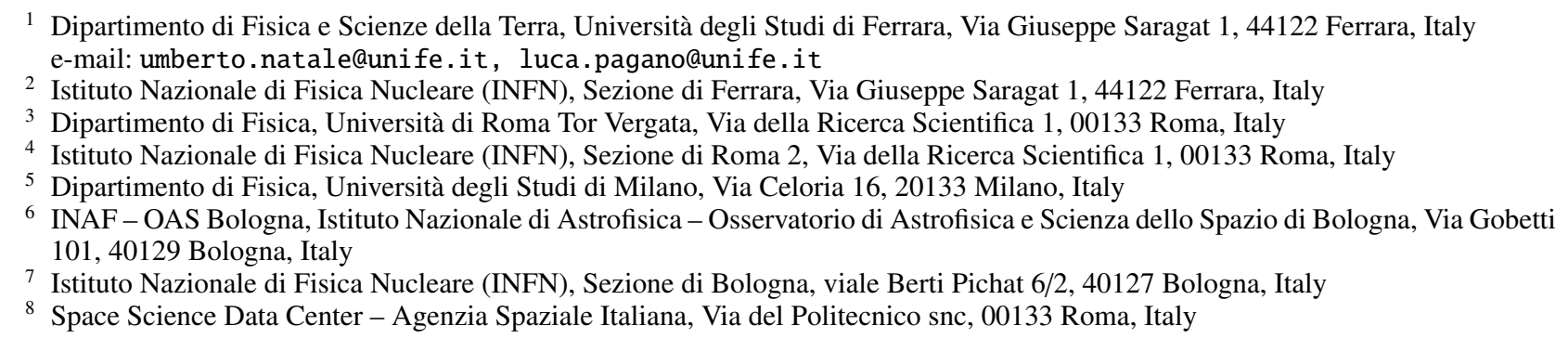

Received 27 May 2020 / Accepted 29 July 2020

\begin{abstract}
We present a cosmic microwave background (CMB) large-scale polarization dataset obtained by combining Wilkinson Microwave Anisotropy Probe (WMAP) in the $K, Q$, and $V$ bands with the Planck $70 \mathrm{GHz}$ maps. We employed the legacy frequency maps released by the WMAP and Planck collaborations and performed our own Galactic foreground mitigation technique, relying on Planck $353 \mathrm{GHz}$ for polarized dust and on Planck $30 \mathrm{GHz}$ and WMAP K for polarized synchrotron. We derived a single, optimally noise-weighted, low residual foreground map and the accompanying noise covariance matrix. These are shown through $\chi^{2}$ analysis to be robust over an ample collection of Galactic masks. We used this dataset, along with the Planck legacy Commander temperature solution, to build a pixel-based low-resolution CMB likelihood package, whose robustness we tested extensively with the aid of simulations, finding an excellent level of consistency. Using this likelihood package alone, we are able to constrain the optical depth to reionization, $\tau=0.069_{-0.012}^{+0.011}$ at $68 \%$ confidence level, on 54\% of the sky. Adding the Planck high- $\ell$ temperature and polarization legacy likelihood, the Planck lensing likelihood, and BAO observations, we find $\tau=0.0714_{-0.0096}^{+0.0087}$ in a full $\Lambda$ CDM exploration. The latter bounds are slightly less constraining than those obtained by employing the Planck High Frequency Instrument's (HFI) CMB data for large-angle polarization, which only include EE correlations. Our bounds are based on a largely independent dataset that includes TE correlations. They are generally compatible with Planck HFI, but lean towards slightly higher values for $\tau$. We have made the low-resolution Planck and WMAP joint dataset publicly available, along with the accompanying likelihood code.
\end{abstract}

Key words. cosmological parameters - dark ages, reionization, first stars

\section{Introduction}

Measuring the cosmic microwave background (CMB) polarization at large angular scales is crucial for constraining the reionization peak and, in particular, for the determination of the Thomson scattering optical depth to reionization, $\tau$, which is currently the less constrained of the $\Lambda \mathrm{CDM}$ parameters. The optical depth, $\tau$, is connected to the integrated amount of free electrons along the line of sight and provides information on how and when the first stars and galaxies formed.

Remarkable advancements have been made in this field over the last 15 years. The Wilkinson Microwave Anisotropy Probe (WMAP) (Hinshaw et al. 2013) and Planck (Planck Collaboration VI 2020) collaborations have continuously improved the quality of large-scale polarization measurements, which are known to be notoriously extremely tough to clean from contaminations coming from the foreground and instrumental systematic effects. The most constraining dataset currently available is provided by the Planck collaboration (Planck Collaboration I 2020), which uses the High Frequency Instrument (HFI) measurements at 100 and $143 \mathrm{GHz}$.
Such results for the Legacy Planck release are presented in Planck Collaboration III (2020) and Planck Collaboration V (2020), while an improved post-Planck analysis is presented in Delouis et al. (2019) and in Pagano et al. (2020).

These HFI-based measurements are all specifically designed to determine the reionization optical depth and, thus, they are mainly dedicated to the characterization of the $E$-modes power spectrum. This approach, which is consistent with the corresponding likelihood codes delivered, is mainly driven by the difficulty of building reliable noise covariance matrices and by the relatively high level of residual systematic effects related to dipole and foreground temperature-to-polarization leakage. Such likelihoods, despite being the most sensitive to date, do not include the TE spectrum Planck Collaboration V (2020). Furthermore, they cannot be adapted to handle nonrotationally invariant cosmologies in a straightforward way and they might need tuned-up simulations for exotic models (see Planck Collaboration V 2020, Sect. 2.2.6).

For the Legacy data release, together with the HFI-based likelihood, the Planck collaboration has also delivered a mapbased likelihood employing observations of the Low Frequency 
Instrument (LFI) in the $70 \mathrm{GHz}$ channel. The sensitivity to the reionization optical depth of the LFI-based likelihood is inferior by more than a factor of two with respect to the HFI-based likelihood.

The possibility of combining the WMAP and Planck observations to build a "joint" dataset that is more constraining was first explored by some of our team in Lattanzi et al. (2017), using the data available at the time. To date, however, a combined dataset using the WMAP and Planck legacy observations of the large-scale polarization is not publicly available. Aiming to fill this gap, here we present a combined real-space polarization dataset which jointly considers the Planck $70 \mathrm{GHz}$ channel and the WMAP $K a, Q$, and $V$ bands. Because of the aforementioned difficulty in dealing with residual systematic effects in the pixel space (Planck Collaboration V 2020), we do not consider the HFI CMB channels, such as 100 and $143 \mathrm{GHz}$.

An important aspect of our work is that we perform an independent analysis pipeline starting from the raw maps, as delivered by the two collaborations. From there, we build polarization masks, perform foreground cleaning through a template fitting and, finally, combine the four maps in the pixel domain through inverse noise weighting. The resulting dataset, despite still having an overall higher noise than the HFI-based one, allows for an independent estimation of the reionization optical depth. Moreover, being a real-space dataset, it is suitable for a number of studies that are not accessible for a spectrumbased likelihood (see, e.g., Planck Collaboration XXIII 2014; Planck Collaboration XVI 2016) and it is capable of exploring non-rotationally invariant cosmologies. For an exhaustive review of likelihood methods, see Gerbino et al. (2020).

The paper is organized as follows. We start by describing the input datasets in Sect. 2. In Sect. 3, we present the new set of masks produced for the Planck and WMAP data. In Sect. 4, we introduce the main algorithms used in the paper, including a detailed discussion of the role of the regularization noise used in the analysis. In Sect. 5, we present the results of the component separation process. In Sects. 6 and 7, we show the angular power spectra and study the stability of the $\tau$ estimates in different masks, in addition to performing a Monte Carlo validation. We present our constraints on the reionization optical depth, $\tau$, in Sect. 8 and we draw our conclusions in Sect. 9.

\section{Datasets}

In this section, we describe the large-scale WMAP and Planck polarization maps that were used to build the combined dataset. As already mentioned, as CMB channels, we considered the $70 \mathrm{GHz}$ channel from Planck LFI (Planck Collaboration II 2020) and the $K a, Q$, and $V$ bands from WMAP (Bennett et al. 2013). In the case of LFI $70 \mathrm{GHz}$, we used the full mission map after removing the bandpass and gain-mismatch-leakage correction maps. These maps, described in Planck Collaboration II (2020), are part of the Planck 2018 legacy data release, and are publicly available through the Planck Legacy Archive ${ }^{1}$. For WMAP, we use the raw nine-year frequency maps, available on the Lambda archive ${ }^{2}$. In principle, we could have also considered the $44 \mathrm{GHz}$ channel from Planck LFI and the $W$-band from WMAP as CMB channels. However, we found that both these channels show excess power, likely to be spurious in origin, after implementing the foreground cleaning procedure described in Sect. 4.

\footnotetext{
1 http://pla.esac.esa.int/pla/

2 https://lambda.gsfc.nasa.gov/product/map/dr5/m_ products.cfm
}

For this reason, we decided not to include the $44 \mathrm{GHz}$ and $W$-band channels in our analysis. We note that the Planck and WMAP collaborations made the same choice on similar grounds (Planck Collaboration V 2020; Page et al. 2007).

We employed the $K$-band from WMAP, LFI $30 \mathrm{GHz}$, and HFI $353 \mathrm{GHz}$ maps from Planck as tracers of Galactic foreground emission. These are used both to generate masks excluding regions dominated by Galactic emissions, and to mitigate the astrophysical foreground contamination in the remaining parts of the sky, as explained in detail in Sects. 3 and 4. At $30 \mathrm{GHz}$, we used the full-mission, bandpass leakage-corrected map. For the $353 \mathrm{GHz}$ channel, we selected a map built only from data provided by polarization-sensitive bolometers (PSB; Planck Collaboration III 2020), as done in the low- $\ell$ analysis presented in Planck Collaboration V (2020). The WMAP $K$ band and Planck $30 \mathrm{GHz}$ are used as a polarized synchrotron tracer, respectively, for the WMAP and Planck CMB channels. This follows the prescription of Lattanzi et al. (2017) and Weiland et al. (2018). The Planck $353 \mathrm{GHz}$ is used as polarized thermal dust tracer for both WMAP and Planck.

Since we are mainly focused on the large angular scales, it appears convenient to work with low-resolution datasets. Thus, all the maps of the Stokes parameters, $\mathbf{m}=[Q, U]$, describing the measured linear polarization, were downgraded to a HEALPix resolution of $N_{\text {side }}=16$ (Górski et al. 2005), which corresponds to a pixel size of $\sim 3.7$ degrees. A smoothing kernel was applied to the high-resolution maps prior to the downgrading, which is meant to avoid aliasing into the large angular scales of the high-frequency power present in the maps. The smoothing was performed in harmonic space, using a cosine window function (Benabed et al. 2009; Planck Collaboration V 2020). This guarantees that the signal is left unaltered on the scales of interest, that is, up to multipoles of $\ell=N_{\text {side }}=16$, while it is smoothly set to zero on smaller scales, $\ell>3 \times N_{\text {side }}=48$.

The instrumental noise properties of each low-resolution map are described by an associated pixel-pixel noise covariance matrix (NCVM). For the LFI channels, the covariance matrices are presented in Planck Collaboration II (2020). The $70 \mathrm{GHz}$ covariance matrix has been rescaled in harmonic space in order to match the noise level of the half-difference of half-ring maps, following the procedure described in Planck Collaboration V (2020). For the HFI $353 \mathrm{GHz}$ NCVM, we use a downgraded version of the map-making covariance matrix, which is instead generated at the native high-resolution of $N_{\text {side }}=2048$. This NCVM only accounts for $\mathrm{Q}$ and $\mathrm{U}$ correlations within the same pixel, while correlations between different pixels are ignored. Finally, for WMAP we build the NCVMs starting from the polarization pixel-pixel inverse covariance matrices at $N_{\text {side }}=16$ (Res 4) delivered by the WMAP team and described in Page et al. (2007), Bennett et al. (2013). The cosine window function apodization is performed in harmonic space on the eigenvectors of these low resolution matrices. It is worth noting that although exchanging the order of the smoothing and downgrading operations is clearly not an option at the map level, due to the possible presence of sub-pixel structure, it can still be acceptable for the NCVMs.

Since all the $(Q, U)$ NCVMs were convolved with a smoothing function, we added to them a white noise covariance matrix, with $\sigma^{2}=(20 \mathrm{nK})^{2}$, in order to guarantee that they are numerically well-conditioned, as in Planck Collaboration V (2020). For consistency, noise with the same statistical properties has to be added to the corresponding maps. However, instead of adding a single noise realization to each smoothed data map, as in Planck Collaboration V (2020), we followed a different 
procedure, which is described in Sect. 4. This ensures that our results are not biased by a particular realization of the regularization noise.

Concerning the temperature $(T)$ map, we always employ the Planck 2018 Commander solution (Planck Collaboration IV 2020) outside its confidence mask, which leaves $86 \%$ of the sky available. This map was filtered with a Gaussian beam of FWHM 440 arcmin and downgraded to $N_{\text {side }}=16$. Since it is reasonable to assume that the temperature noise at large angular scales is negligible, we only need to include the regularization noise. Thus, we modeled the temperature NCVM as a white noise covariance matrix with $\sigma^{2}=(2 \mu \mathrm{K})^{2}$, as in Planck Collaboration V (2020). We consistently handle such regularization noise following the same procedure adopted for polarization. Finally, when building the NCVM of the full TQU maps, we neglect the correlation between temperature and polarization and set the corresponding off-diagonal blocks in the covariance matrix to zero (Planck Collaboration XI 2016).

\section{Polarization masks}

In order to efficiently perform the foreground cleaning and the cosmological parameter estimation, we must remove the pixels of the data map that are most affected by foreground contamination from the analysis. With regard to temperature, we always use the Commander 2018 confidence mask (Planck Collaboration V 2020) provided by the Planck collaboration. In this section, we describe how the polarization masks are produced.

In polarization, we built two different sets of masks for WMAP and LFI. For LFI $70 \mathrm{GHz}$, we used the 30 and the $353 \mathrm{GHz}$ maps as, respectively, synchrotron $(s)$ and dust $(d)$ tracers, analagously to what is done in Planck Collaboration V (2020). We first applied a Gaussian smoothing with a full width half maximum (FWHM) of $7.5^{\circ}$ to these input maps, taken at their native resolution of $N_{\text {side }}=1024(30 \mathrm{GHz})$ and $N_{\text {side }}=$ $2048(353 \mathrm{GHz})$. Then we built maps of the polarization amplitude $P_{\mathrm{s}}=\alpha \sqrt{Q_{\mathrm{s}}^{2}+U_{\mathrm{s}}^{2}}$ and $P_{\mathrm{d}}=\beta \sqrt{Q_{\mathrm{d}}^{2}+U_{\mathrm{d}}^{2}}$, where the scaling coefficients are set to $\alpha=0.063$ and $\beta=0.0077$, as estimated in Planck Collaboration XI (2016). These two maps were subsequently downgraded to the HEALPix resolution $N_{\text {side }}=16$. From these maps, two separate sets of masks for synchrotron and dust emission were built as follows. We excluded pixels where the relevant polarization intensity, $P_{\mathrm{s}}$ or $P_{\mathrm{d}}$, is greater than a given threshold. This threshold is expressed in terms of excess intensity with respect to the corresponding mean value, $\left\langle P_{\mathrm{s}}\right\rangle$ or $\left\langle P_{\mathrm{d}}\right\rangle$, over the whole sky. Any pair of synchrotron and dust masks can then be combined to yield a single foreground mask. Varying the threshold, we were able to build foreground masks keeping a chosen fraction of the sky. We chose to build, for LFI $70 \mathrm{GHz}$, nine different masks with equally spaced sky fractions $f_{\text {sky }}=30 \%, 35 \%, \ldots, 65 \%, 70 \%$. We did not consider larger sky fractions because, as we show in Sect. 5, we find an indication of excess residual power in the LFI maps after foreground removal for masks with $f_{\text {sky }}>60 \%$. A subset of the LFI masks is shown in Fig. 1.

A corresponding set of masks for WMAP channels is built through a similar procedure. Here, we use the WMAP $K$-band as a tracer for synchrotron emission and Planck $353 \mathrm{GHz}$ for dust. These are rescaled using the coefficients in Lattanzi et al. (2017); for completeness, these values are also reported in Table 1 here. In this case, when the mask structure at intermediate and high latitudes is dominated by the synchrotron emission (i.e., by the $K$-band). Thus we decided to adopt the same mask for the three

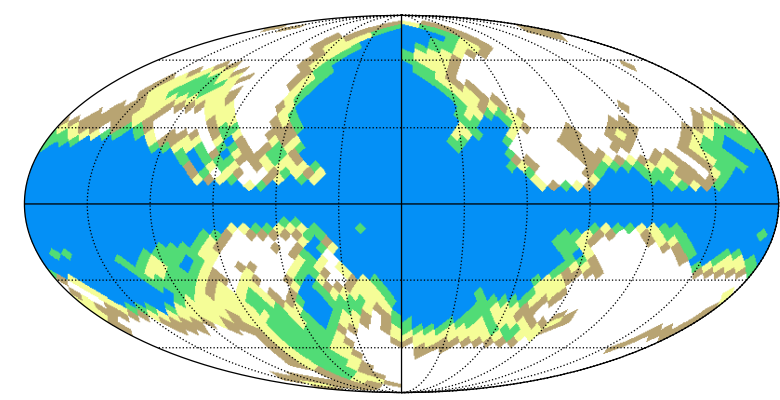

Fig. 1. Subset of the masks used in the analysis of the LFI $70 \mathrm{GHz}$ data. Values of the available sky fraction $f_{\text {sky }}$ in each mask are $30 \%, 40 \%$, $50 \%$, and $60 \%$.

Table 1. Foreground scalings coefficients from WMAP $K$-band $(\alpha)$ and Planck $353 \mathrm{GHz}(\beta)$ to the indicated WMAP channels.

\begin{tabular}{lcc}
\hline \hline \multicolumn{1}{c}{ Channel } & $\alpha$ & $\beta$ \\
\hline$K a$ band & 0.315 & 0.0031 \\
$Q$ band & 0.163 & 0.0039 \\
$V$ band & 0.047 & 0.0076 \\
\hline
\end{tabular}

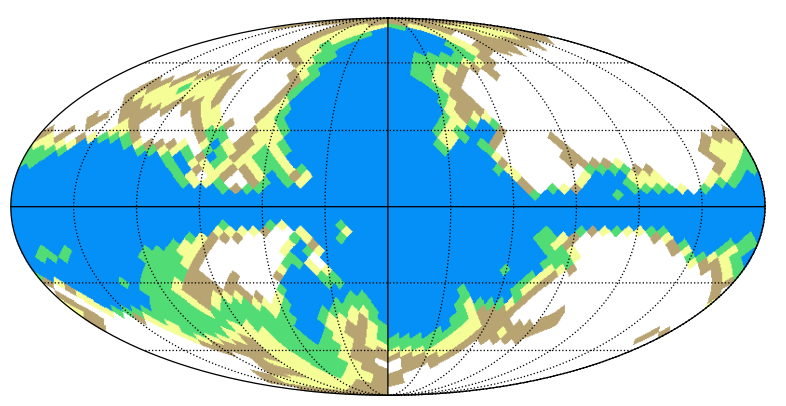

Fig. 2. Subset of the masks used in the analysis of the WMAP data. Values of the available sky fraction $f_{\text {sky }}$ in each mask are $30 \%, 40 \%$, $50 \%$, and $60 \%$.

WMAP bands. This leads to a single set of ten masks, with a sky fraction ranging from $30 \%$ to $75 \%$ in steps of $5 \%$. A subset of the masks is shown in Fig. 2.

Finally, with the aim of building a WMAP-Planck LFI combined dataset, we also produced another set of masks to be used in the analysis of the joint dataset. These were built by combining pairs of WMAP and LFI masks, taking the pixels that are left available in at least one of the two masks. In other words, if we think of a mask as the set of all pixels that can be used in the analysis, the "joint" masks are the union (in the set-theory meaning of the word) of the individual WMAP and LFI masks. For this reason, the sky fraction of each combined mask is always equal or larger than the sky fractions of the individual masks it is built from. For example, the union of the WMAP and Planck LFI $30 \%$ masks has $f_{\text {sky }} \simeq 35 \%$. We then chose to produce a set of ten masks built as follows. The first seven masks are the union of each pair of WMAP and Planck masks with the same sky fraction $f_{\text {sky }}=30 \%, 35 \%, \ldots, 55 \%, 60 \%$. The remaining three masks are the union of the LFI $60 \%$ mask with the WMAP $65 \%, 70 \%$ and $75 \%$ masks. The reason behind this choice is, as mentioned above and discussed in more detail in Sect. 6, that we do not consider the LFI masks with $f_{\text {sky }}>60 \%$ to be suitable for cosmological analyses. The sky fractions for the set of union masks, together with the individual masks used to produce them, are summarized in Table 2. 
Table 2. Masks used in the analysis of the joint WMAP-Planck dataset.

\begin{tabular}{lc}
\hline \hline Individual $f_{\text {sky }}($ WMAP $\times$ Planck LFI $)$ & Total $f_{\text {sky }}$ \\
\hline $30 \% \times 30 \%$ & $35 \%$ \\
$35 \% \times 35 \%$ & $40 \%$ \\
$40 \% \times 40 \%$ & $45 \%$ \\
$45 \% \times 45 \%$ & $50 \%$ \\
$50 \% \times 50 \%$ & $54 \%$ \\
$55 \% \times 55 \%$ & $59 \%$ \\
$60 \% \times 60 \%$ & $63 \%$ \\
$65 \% \times 60 \%$ & $66 \%$ \\
$70 \% \times 60 \%$ & $70 \%$ \\
$75 \% \times 60 \%$ & $75 \%$ \\
\hline
\end{tabular}

Notes. Each mask is built as the union of the individual masks, reported in the left column, and leaves the sky fraction reported on the right available for analysis.

\section{Methods}

In this section, we describe the cleaning procedure and the likelihood approximation used in cosmological parameter estimation. We pay particular attention to the impact of regularization noise on both scalings and cosmological parameters estimation and, at the end of the section, we discuss how it can be mitigated.

The cleaning procedure adopted here is based on fitting foreground templates at the map level (see, e.g., Page et al. 2007; Planck Collaboration XI 2016; Planck Collaboration V 2020). Denoting the linear polarization map at a given frequency, $v$, with $\mathbf{m}_{v}^{P}=\left[\mathbf{Q}_{v}, \mathbf{U}_{v}\right]$, the corresponding foreground-cleaned map $\widetilde{\mathbf{m}}_{v}^{P, \mathrm{fc}}$ is ${ }^{3}$

$\widetilde{\mathbf{m}}_{v}^{P, \mathrm{fc}}=\frac{\mathbf{m}_{v}^{P}-\alpha_{v} \mathbf{t}^{\mathrm{s}}-\beta_{v} \mathbf{t}^{\mathrm{d}}}{1-\alpha_{v}-\beta_{v}}$,

where $\mathbf{t}^{\mathrm{s}}\left(\mathbf{t}^{\mathrm{d}}\right)$ and $\alpha_{v}\left(\beta_{v}\right)$ are the tracers and the scaling coefficient for synchrotron (dust) emission, respectively, described in Sect. 2. Thus, if $S^{P}$ and $N_{v}^{P}$ are, respectively, the signal and noise covariance matrices at frequency, $v$, the fitted coefficients in Eq. (1) are estimated by minimization of the quantity:

$\chi_{v}^{2}=\left(\widetilde{\mathbf{m}}_{v}^{P, \mathrm{fc}}\right)^{T} \widetilde{\mathrm{C}}_{v}^{-1} \widetilde{\mathbf{m}}_{v}^{P, \mathrm{fc}}$,

where $\widetilde{\mathrm{C}}_{v} \equiv\left\langle\widetilde{\mathbf{m}}_{v}^{P \text { fc }}\left(\widetilde{\mathbf{m}}_{v}^{P, \mathrm{fc}}\right)^{\top}\right\rangle$ is the covariance matrix,

$\widetilde{C}_{v}=S^{P}\left(C_{\ell}^{\mathrm{fid}}\right)+\frac{N_{v}^{P}+\alpha_{v}^{2} N^{s}+\beta_{v}^{2} N^{d}}{\left(1-\alpha_{v}-\beta_{v}\right)^{2}}$.

We note that $\chi_{v}^{2}$ is a $\chi^{2}$-distributed quantity when considered as a function of the map but not as a function of the scalings.

Here, $N^{s}$ and $N^{d}$ are the polarization parts of the NCVMs for the foregrounds tracers. The signal covariance matrix is built as described in Tegmark \& de Oliveira-Costa (2001) and assumes a fiducial power spectrum, $C_{\ell}^{\text {fid }}$, taken as the Planck legacy best-fit (Planck Collaboration VI 2020). The inversion of $\widetilde{C}_{\nu}$, needed to compute the $\chi^{2}$ in Eq. (2), requires the addition of some regularization noise. In particular, we follow the approach used in the Planck legacy analysis (Planck Collaboration XI 2016; Planck Collaboration VI 2020) and consider white noise in polarization with $\mathrm{rms} \sigma_{r}^{P}=20 \mathrm{nK}$. We thus sum a random white noise realization, $\mathbf{n}_{r}^{P}$, with this amplitude to $\widetilde{\mathbf{m}}_{v}^{P \text {,fc }}$ and add a diagonal term, $N_{r}^{P} \equiv\left(\sigma_{r}^{P}\right)^{2} \mathbf{I}$, to the covariance matrix (3) and

\footnotetext{
3 Here "fc" stands for "foreground-cleaned".
}

then use these regularized objects to build the $\chi^{2}$ in Eq. (2). In the following, we denote the cleaned map with regularization noise added as $\mathbf{m}_{v}^{P \text { ffc }} \equiv \widetilde{\mathbf{m}}_{v}^{P \text {,fc }}+\mathbf{n}_{r}^{P}$ and the associated covariance matrix as $\mathrm{C}_{v} \equiv\left\langle\mathbf{m}_{v}^{P, \mathrm{fc}}\left(\mathbf{m}_{v}^{P, \mathrm{fc}}\right)^{\top}\right\rangle=\widetilde{\mathrm{C}}_{v}+N_{r}^{P}$.

Once $\alpha_{v}$ and $\beta_{v}$ have been estimated through this minimization procedure, we can define the cleaned data vector $\mathbf{m}_{v}^{\text {fc }} \equiv\left[\mathbf{T}, \mathbf{m}_{v}^{P \text {,fc }}\right]$, with $\mathbf{T}$ being the Commander map described in Sect. 2. We write down its likelihood function (Gerbino et al. 2020), $\mathcal{L}\left(C_{\ell}\right) \equiv P\left(\mathbf{m}_{v}^{\mathrm{fc}} \mid C_{\ell}\right)$, as

$$
\begin{aligned}
- & 2 \log \mathcal{L}\left(C_{\ell}\right)=\log \left|S\left(C_{\ell}\right)+N_{v}^{\mathrm{fc}}\right| \\
& +\left(\mathbf{m}_{v}^{\mathrm{fc}}\right)^{\top}\left(S\left(C_{\ell}\right)+N_{v}^{\mathrm{fc}}\right)^{-1} \mathbf{m}_{v}^{\mathrm{fc}}+\text { const. }
\end{aligned}
$$

The NCVM $N_{v}^{\mathrm{fc}}$ used in the likelihood analysis is built as follows. The $T T$ block is consistent with the Commander map having only white regularization noise with $\mathrm{rms} \sigma_{r}^{T}=2 \mu \mathrm{K}$, while the $T Q$ and $T U$ blocks are vanishing. The polarization part $N_{v}^{P, f c}$ of the NCVM is instead given by

$$
N_{v}^{P, \mathrm{fc}}=\frac{N_{v}^{P}+\alpha_{v}^{2} N_{\mathrm{s}}+\beta_{v}^{2} N_{\mathrm{d}}+\sigma_{\alpha_{v}}^{2} \mathbf{t}^{\mathrm{s}}\left(\mathbf{t}^{\mathrm{s}}\right)^{\top}+\sigma_{\beta_{v}}^{2} \mathbf{t}^{\mathrm{d}}\left(\mathbf{t}^{\mathrm{d}}\right)^{\top}}{\left(1-\alpha_{v}-\beta_{v}\right)^{2}}+N_{r}^{P},
$$

where $\sigma_{\alpha_{v}}$ and $\sigma_{\beta_{v}}$ are the uncertainties in the estimates of foreground scaling coefficients and $\mathbf{t}^{\mathrm{s}, \mathrm{d}}\left(\mathbf{t}^{\mathrm{s}, \mathrm{d}}\right)^{\top}$ is the outer product of the tracer maps.

The addition of regularization noise has a small, but not completely negligible, impact on the determination of the foreground scaling coefficients, and, consequently, on cosmological parameter estimates. In fact, the extra noise added to the map increases the scatter of point estimates (e.g., the posterior mean) of parameter values around the true value. Moreover, the extra term added to the NCVM increases parameter uncertainties. In what follows, we first assess the magnitude of the former effect at the level of both scaling coefficients and cosmological parameters. We then illustrate how we manage to avoid extracting a particular noise realization, which leads to non-negligible scatter (as compared to the one caused by instrumental noise).

In order to show and quantify the extra scatter in the estimates of $\alpha, \beta$ and cosmological parameters induced by regolarization noise, we proceed as follows. We draw 1000 white noise realizations, $\mathbf{n}_{r, i}(i=1, \ldots, 1000)$, with an rms of $2 \mu \mathrm{K}$ in temperature and $20 \mathrm{nK}$ in polarization. We then estimate $\alpha$ and $\beta$ on the Planck $70 \mathrm{GHz}$ channel, following the procedure illustrated above, using each of the realization just described as the regularization noise map. For the sake of this test, we adopt a mask that retains $50 \%$ of the sky. This procedure results in 1000 Monte Carlo estimates, $\alpha_{i}$ and $\beta_{i}$. Once the scaling coefficients have been obtained, we further proceed with an estimation of the cosmological parameters $\left(\log \left(10^{10} A_{s}\right)\right)_{i}$ and $\tau_{i}$ from the likelihood in Eq. (4). We note that in this last step, we consistently use the same regularization noise used when fitting the scaling coefficients.

Since the CMB signal and the instrumental noise are the same in each map belonging to this ensemble, the scatter in the recovered values of the parameters provides an estimate of the dependence on the regularization noise realization, at the level of both scalings and cosmological parameters. The results of this procedure are shown in Fig. 3, where we show the distribution of the $\alpha_{i}$ 's, $\beta_{i}$ 's, and $\tau_{i}$ 's with respect to the mean value, in units of the average uncertainty. We also show the $\chi^{2}$ computed from Eq. (2) in units of $\sigma_{\chi^{2}}=\sqrt{2 N_{\text {d.o.f. }}}$. 


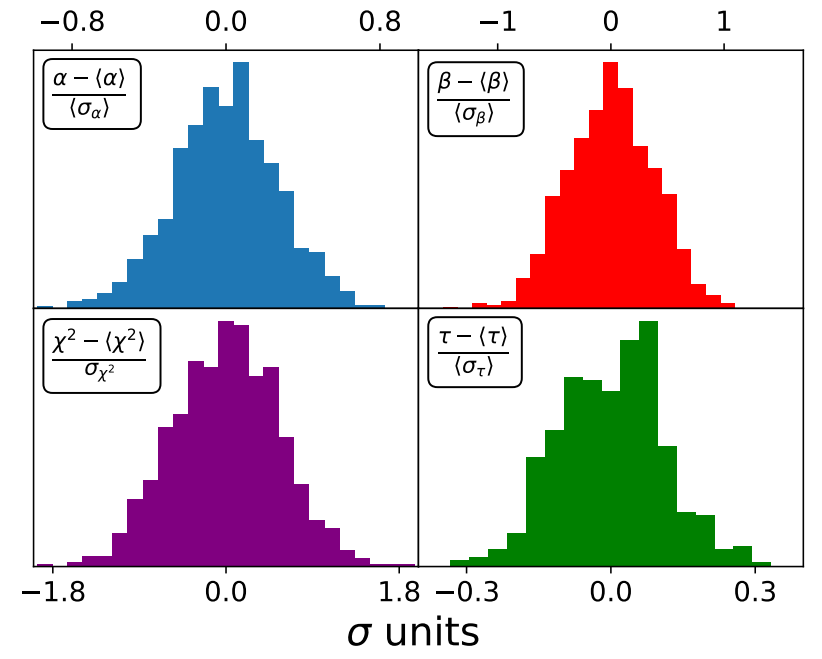

Fig. 3. Histograms of the expected scatter in the recovered foreground scalings, in the $\chi^{2}$ of the component separation and in the measured $\tau$ due to the regularization noise. For each quantity, we show the distance from the center of the empirical distribution in units of $\sigma$.

We then compute their standard deviation, that is, $\sqrt{\left\langle(\theta-\langle\theta\rangle)^{2}\right\rangle} /\left\langle\sigma_{\theta}\right\rangle$, where $\theta=\left\{\alpha, \beta, \chi^{2}, \tau\right\}$. For the synchrotron scaling coefficient, $\alpha$, the scatter induced by the extra noise is, on average, 0.27 times the average parameter uncertainty. In other words, $68 \%$ of the $\alpha_{i}$ deviates from $\langle\alpha\rangle$ by less than $0.27 \times\left\langle\sigma_{\alpha}\right\rangle$. The corresponding value for $\beta$ is 0.38 times the average parameter uncertainty. The $\chi^{2}$ of the cleaned map is the most affected quantity by the particular realization of regularization noise. In fact, the impact is, at the $1 \sigma$ level, at most 0.55 times the expected width of a $\chi^{2}$ distribution with $N_{\text {d.o.f. degrees of free- }}$ dom. This extra scatter in the scaling estimates induces a smaller, but still non-negligible, effect on the final $\tau$ determination. The effect on $\tau$, at one standard deviation of the distribution, equals $11 \%$ of its average uncertainty.

Thus, when we add regularization noise, we pay the price of an increased parameter uncertainty, and we might also be prone to unwanted parameter shifts caused by an unlucky choice of the actual noise realisation used. For example, a 3- $\sigma$ noise realization can easily shift the scalings by $\sim 1 \sigma$ and $\tau$ by $0.3 \sigma$. In fact roughly $1 \%$ of the noise realizations in our Monte Carlo resulted in shifts larger than 1 and $0.3 \sigma$ 's for the scalings and $\tau$, respectively.

A possible way to avoid large parameter shifts is to somehow average over different realizations of the regularization noise. In order to do so, we draw $N_{i t}=1000$ white noise realizations $\mathbf{n}_{r, i}$ $(i=1, \ldots, 1000)$ with $20 \mathrm{nK}$ rms. For given values of $\alpha$ and $\beta$, these are used to build as many cleaned polarization maps $\mathbf{m}_{i}^{P \text {,fc }}=\widetilde{\mathbf{m}}^{P \text {,fc }}+\mathbf{n}_{\mathrm{r}, i}$ and the following quantity:

$\overline{\chi^{2}}=\frac{1}{N_{i t}} \sum_{i=1}^{N_{i t}}\left(\mathbf{m}_{i}^{P, \mathrm{fc}}\right)^{\top} C^{-1} \mathbf{m}_{i}^{P, \mathrm{fc}}$.

We note that $\overline{\chi^{2}}$ does not follow a chi-square distribution. It is straightforward to show that its expectation value over the regularization noise is

$\left\langle\overline{\chi^{2}}\right\rangle_{\mathbf{n}_{r}}=\left(\widetilde{\mathbf{m}}^{P, \mathrm{fc}}\right)^{\top} C^{-1} \widetilde{\mathbf{m}}^{P, \mathrm{fc}}+\operatorname{Tr}\left(C^{-1} N_{r}^{P}\right)$,

which is the same as the expectation value of the $\chi^{2}$ built from a single regularized map, $\chi^{2}=\left(\mathbf{m}^{P, \mathrm{fc}}\right)^{\top} C^{-1} \mathbf{m}^{P, \mathrm{fc}}$. Also, this expec- tation value is different from the value of the $\chi^{2}$ on the regularization noise-free map,

$\left(\widetilde{\mathbf{m}}^{P, \mathrm{fc}}\right)^{T} \widetilde{C}^{-1} \widetilde{\mathbf{m}}^{P, \mathrm{fc}}$. The variance associated to $\overline{\chi^{2}}$ is:

$\operatorname{Var}\left[\overline{\chi^{2}}\right]_{\mathbf{n}_{r}}=\frac{1}{N_{i t}}\left\{4\left(\widetilde{\mathbf{m}}^{P, \mathrm{fc}}\right)^{\top} \mathrm{C}^{-1} N_{\mathrm{r}}^{P} \mathrm{C}^{-1} \widetilde{\mathbf{m}}^{P, \mathrm{fc}}+2 \operatorname{Tr}\left[\left(\mathrm{C}^{-1} N_{\mathrm{r}}^{P}\right)^{2}\right]\right\}$

that, as should be expected, goes to 0 as the number of noise realizations, over which the average is performed, increases.

For these reasons, we chose to minimize the quantity in Eq. (6) to obtain estimates of the scaling coefficients that are less dependent on the particular realization of regularization noise. Similarly, when estimating cosmological parameters, we performed an analogous procedure by drawing $N_{i t}=1000$ noise realizations in temperature and polarization, and using the average of the quantity defined in Eq. (4) over these realizations. The results of these procedures are presented in the next sections.

\section{Foreground cleaning}

In this section, we discuss the results of the estimation of the syncrotron and dust scaling coefficients for the different channels in various masks. We also discuss how this leads to the choice of the "confidence" masks that are used to produce the foregroundcleaned maps for each channel and how inverse-noise-weighted combinations of these maps are built.

We clean independently the four cosmological channels (i.e., WMAP $K a, Q$ and $V$ bands and Planck $70 \mathrm{GHz}$ ), following the template-fitting procedure described in Sect. 4. We thus minimize Eq. (6) to estimate the synchrotron, $\alpha_{v}$, and dust, $\beta_{v}$, scaling coefficients for each map. The final polarization map, $\widetilde{\mathbf{m}}^{\text {Pfc }}$, and polarization noise covariance matrix, $N^{P, \text { fc }}$, are given by Eqs. (1) and (5).

Figures 4-7 show the scaling coefficients computed for each cosmological channel in the masks described in Sect. 3. In the bottom panel of each figure, we also show the excess $\chi^{2}$ in units of the expected dispersion, $\sqrt{2 N_{\text {d.o.f. }}}$, that is: $\Delta \chi^{2}=$ $\left(\chi_{v}^{2}-N_{\text {d.o.f. }}\right) / \sqrt{2 N_{\text {d.o.f. }}}$, where $\chi_{v}^{2}$ is computed from Eq. (2).

We use the $\Delta \chi^{2}$ values to select the processing mask to be used in the template fitting. The rule of thumb is to use the mask with the largest $f_{\text {sky }}$ among those with $\Delta \chi^{2} \leq 2$. The only exception is represented by WMAP $K a$ band which shows a sudden change in both scalings, as well an noticeable increase in the excess $\chi^{2}$ between the $55 \%$ and $60 \%$ masks (see Fig. 4). In this case, we cautiously choose the to use the $55 \%$ mask, even though the excess $\chi^{2}$ itself remains slightly below 2 also in the $60 \%$ mask. We note how similar jumps between the 55\% and $60 \%$ masks are evident also in the scalings of the WMAP $Q$ band, shown in Fig. 5. An interesting case is represented by WMAP $V$ band (Fig. 6), for which the $\Delta \chi^{2}$ reaches a maximum in the $55 \%$ mask before decreasing for larger masks, without ever reaching the threshold $\Delta \chi^{2}=2$. In this case we chose the $75 \%$ mask. The masks used in the foreground cleaning, together with the scaling coefficients obtained, are reported in Table 3.

The resulting cleaned maps can then be combined together to build inverse-noise-weighted maps. In particular, we build two combinations: the first is a "WMAP-only" map built from the $K a, Q$, and $V$ bands, while the second is a joint "WMAP+LFI" map, that uses the $K a, Q$, and $V$ WMAP bands and Planck LFI $70 \mathrm{GHz}$ (hereafter WMAP+LFI).

To provide more detail, if $\widetilde{\mathbf{m}}_{v}^{P \text {,fc }}$ is the final cleaned map of the band, $v$, with corresponding noise-covariance matrix, $N_{v}^{P \text {,fc }}$, 


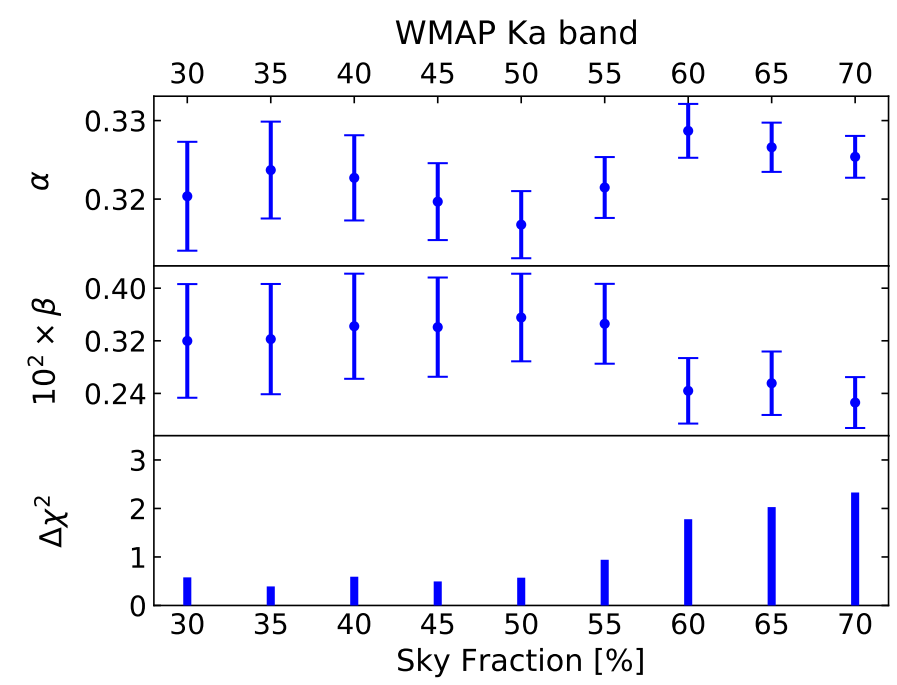

Fig. 4. Scaling coefficients for synchrotron and dust (top and middle panels) estimated on different masks for WMAP $\mathrm{Ka}$ band. Bottom panel: excess $\chi^{2}$ in units of $\sqrt{2 N_{\text {d.o.f. }}}$, i.e., $\Delta \chi^{2}=\left(\chi^{2}-N_{\text {d.o.f. }}\right) / \sqrt{2 N_{\text {d.o.f. }}}$.

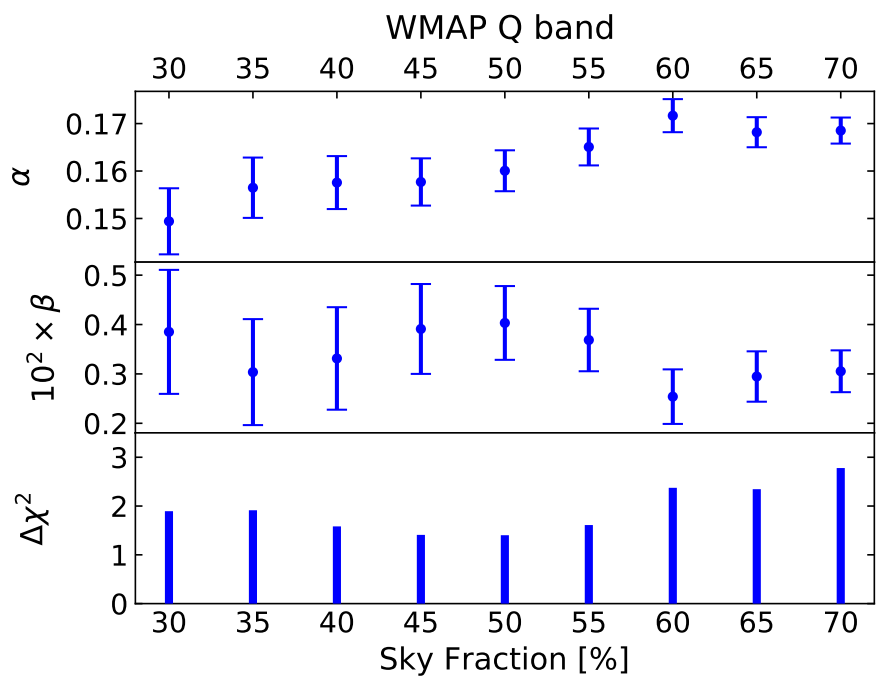

Fig. 5. Same as Fig. 4, but for the WMAP $Q$ band.

the final noise weighted map, $\mathbf{m}^{\mathrm{nw}}$, is built as:

$$
\begin{aligned}
\mathbf{m}^{\mathrm{nw}} & =\left[\sum_{v}\left(N_{v}^{P, \mathrm{fc}}\right)^{-1}\right]^{-1} \sum_{v}\left(N_{v}^{P, \mathrm{fc}}\right)^{-1} \widetilde{\mathbf{m}}_{v}^{P, \mathrm{fc}} \\
& =\overline{\mathcal{N}} \sum_{v}\left(N_{v}^{P, \mathrm{fc}}\right)^{-1} \widetilde{\mathbf{m}}_{v}^{P, \mathrm{fc}},
\end{aligned}
$$

where we define the total noise covariance matrix:

$\overline{\mathcal{N}}=\left[\sum_{v}\left(N_{v}^{P, \mathrm{fc}}\right)^{-1}\right]^{-1}$.

We note that in Eq. (9), we use the $\widetilde{\mathbf{m}}_{v}^{P \text {,fc }}$ with no regularization noise. This is because we do not want to "bring" the regularization noise into the noise-weighted map as we want to avoid possible biases in parameter estimates induced by particular realizations of the regularization noise, as explained in Sect. 4. However, we are forced to use the covariance matrices $N_{v}^{P \text {,fc }}$ that do include regularization noise since, otherwise, we would not be

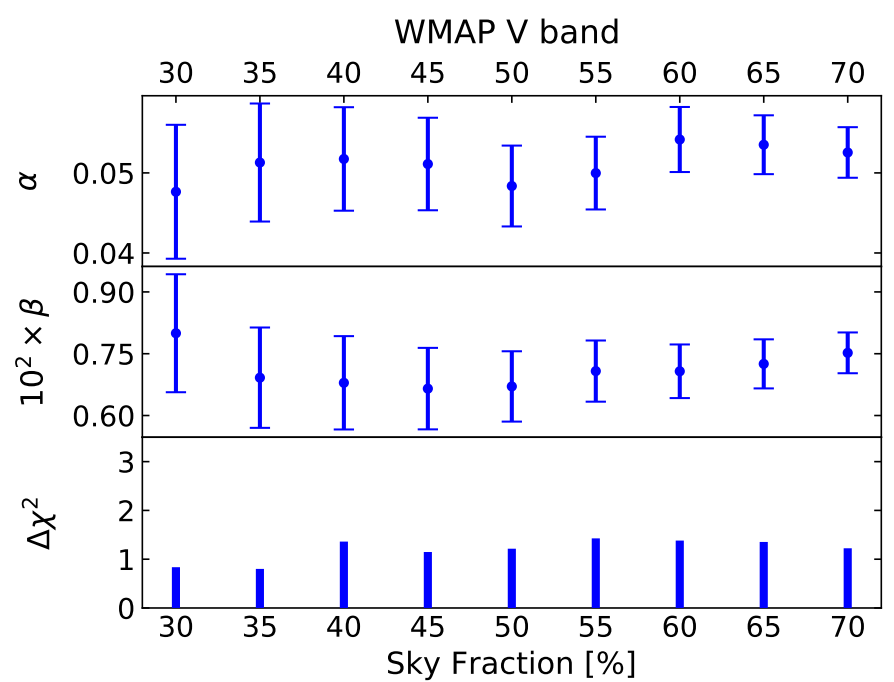

Fig. 6. Same as Fig. 4, but for the WMAP $V$ band.

LFI $70 \mathrm{GHz}$

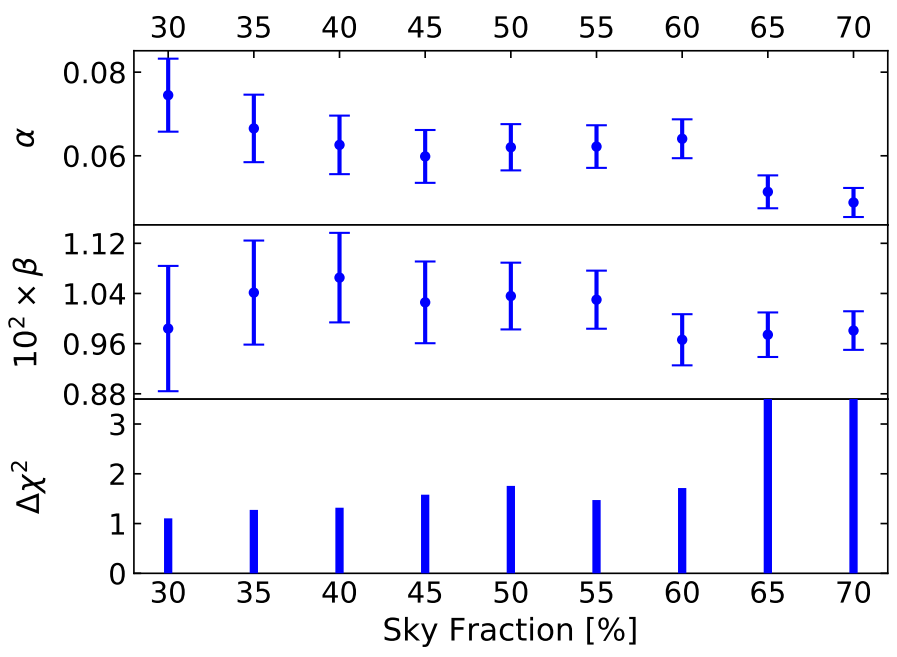

Fig. 7. Same as Fig. 4, but for the Planck LFI $70 \mathrm{GHz}$ channel.

Table 3. Masks used to produce foreground-cleaned maps for each channel, and the corresponding estimates for the scaling coefficients.

\begin{tabular}{lccc}
\hline \hline \multicolumn{1}{c}{ Channel } & Mask & $\alpha$ & $\beta$ \\
\hline$K a$ band & $55 \%$ & $0.3215 \pm 0.0039$ & $0.00346 \pm 0.00061$ \\
$Q$ band & $55 \%$ & $0.1651 \pm 0.0039$ & $0.00369 \pm 0.00063$ \\
$V$ band & $75 \%$ & $0.0527 \pm 0.0027$ & $0.00744 \pm 0.00043$ \\
$70 \mathrm{GHz}$ & $60 \%$ & $0.0641 \pm 0.0046$ & $0.00966 \pm 0.00041$ \\
\hline
\end{tabular}

able to invert them. For this reason, it is evident that $\overline{\mathcal{N}}$ would be the NCVM of a noise-weighted combination built from the (un-tilded) $\mathbf{m}_{v}^{P \text {,fc }}$, but is not the NCVM of $\mathbf{m}^{\text {nw }}$. The actual NCVM can be computed by rewriting Eq. (9) as

$\mathbf{m}^{\mathrm{nw}}=\overline{\mathcal{N}} \sum_{v}\left(N_{v}^{P, \mathrm{fc}}\right)^{-1}\left(\mathbf{s}+\mathbf{n}_{v}^{t}-\mathbf{n}_{v}^{r}\right)$

where $\mathbf{n}_{v}^{t}$ denotes the total noise, i.e., the sum of instrumental and regularization noise, at each frequency. Taking the expectation 
value of $\mathbf{m}^{\mathrm{nw}}\left(\mathbf{m}^{\mathrm{nw}}\right)^{T}$ yields

$$
\begin{aligned}
& \left\langle\mathbf{m}^{\mathrm{nw}}\left(\mathbf{m}^{\mathrm{nw}}\right)^{T}\right\rangle \\
& =S+\overline{\mathcal{N}} \sum_{v v^{\prime}}\left(N_{v}^{P, \mathrm{fc}}\right)^{-1}\left\langle\left(\mathbf{n}_{v}^{t}-\mathbf{n}_{v}^{r}\right)\left(\mathbf{n}_{v^{\prime}}^{t}-\mathbf{n}_{v^{\prime}}^{r}\right)^{T}\right\rangle\left(N_{v^{\prime}}^{P, \mathrm{fc}}\right)^{-1} \overline{\mathcal{N}} \\
& =S+\overline{\mathcal{N}}-\left(\sigma_{r}^{P}\right)^{2} \overline{\mathcal{N}} \sum_{v}\left[\left(N_{v}^{P, \mathrm{fc}}\right)^{-1}\right]^{2} \overline{\mathcal{N}},
\end{aligned}
$$

where we are assuming that the regularization noise $\operatorname{rms} \sigma_{r}^{P}$ for all the involved maps is the same. In the last equation, we use the fact that $\left\langle\mathbf{n}_{v}^{t} \mathbf{n}_{v^{\prime}}^{r}\right\rangle=\left\langle\mathbf{n}_{v}^{r} \mathbf{n}_{v^{\prime}}^{r}\right\rangle=\left(\sigma_{r}^{P}\right)^{2} \mathbf{I} \delta_{v v^{\prime}}$. Thus, the final noise covariance matrix of the combined instrumental noise is

$\mathcal{N} \equiv \overline{\mathcal{N}}-\left(\sigma_{r}^{P}\right)^{2} \overline{\mathcal{N}} \sum_{v}\left[\left(N_{v}^{P, \mathrm{fc}}\right)^{-1}\right]^{2} \overline{\mathcal{N}}$

\section{Power spectra}

In this section, we present our results for the angular power spectra of the maps described in the previous sections. In particular, we use a QML code (Tegmark 1996; Tegmark \& de Oliveira-Costa 2001) to extract the auto power spectra of the cleaned maps described in Sect. 5. In our analysis, power spectra are not directly used for the cosmological parameter extraction. We mainly use them as a probe of possible residual systematics in the maps and, consequently, for selecting the masks suitable for the likelihood analysis. The main tool for performing these consistency tests is the $\chi^{2}$ in harmonic space, defined as:

$\chi_{\mathrm{h}}^{2}=\sum_{\ell, \ell^{\prime}=2}^{\ell_{\max }}\left(C_{\ell}-C_{\ell}^{t h}\right) \mathrm{M}_{\ell \ell^{\prime}}^{-1}\left(C_{\ell^{\prime}}-C_{\ell^{\prime}}^{t h}\right)$,

where $C_{\ell}$ is the power spectrum estimated from a given mapand-mask combination, $\mathrm{M}_{\ell \ell^{\prime}}^{-1}$ is the Fisher matrix and $C_{\ell}^{\text {th }}$ is the power spectrum of a fiducial $\Lambda \mathrm{CDM}$ model with optical depth of $\tau=0.065$ and logarithmic amplitude of primordial scalar fluctuations of $\ln \left(10^{10} A_{s}\right)=3.0343$. We perform separate tests for the $T E, T B, E E, E B$, and $B B$ power spectra. The quantity in Eq. (14) can be compared to the $\chi^{2}$ distribution with $\ell_{\max }-1$ degrees of freedom, computing the corresponding probabilityto-exceed (hereafter PTE). In Tables 4-6, we report the PTEs for LFI, WMAP and WMAP+LFI for different sky fractions, corresponding to the masks presented in Sect. 3. As explained in that section, for the WMAP+LFI dataset, the masks are obtained by combining the individual LFI and WMAP masks. We refer the reader to Table 2 for further details.

Here, we consider $2 \leq \ell \leq 10$, which corresponds roughly to the multipole range affected by the reionization feature. For WMAP, the PTEs are nicely compatible with the theoretical model for all the sky fractions considered. For the LFI dataset we see $\sim 2 \sigma$ deviations for the BB spectrum for intermediate sky fractions $\left(f_{\text {sky }}=40 \%\right.$ and $\left.f_{\text {sky }}=45 \%\right)$, fluctuations reabsorbed in larger sky fractions. In the WMAP+LFI dataset, we do not see any particular failure in the PTEs. We define a "failure" as a PTE $<1 \%$.

We further perform additional consistency tests for the combined dataset. We compute the PTEs for different choices of $\ell_{\max }$, exploring the $\chi^{2}$ consistency up to $\ell=15$ and $\ell=29$. For all the sky fraction we have considered, we do not observe any failure in the total PTEs as a function of $\ell_{\max }$. We also compute the $\ell$-by- $\ell$ PTEs for all the polarization power spectra. The mask keeping a $54 \%$ fraction of the whole sky has the lowest number of outliers
Table 4. Probability to exceed $\chi_{h}^{2}$ for LFI $70 \mathrm{GHz}$ as a function of the sky fraction.

\begin{tabular}{lccccc}
\hline \hline & \multicolumn{5}{c}{ PTE [\%] } \\
\cline { 2 - 6 } Sky fraction & TE & EE & BB & TB & EB \\
\hline $30 \%$ & 62.2 & 87.7 & 16.0 & 56.2 & 74.2 \\
$35 \%$ & 49.9 & 75.3 & 38.5 & 32.8 & 51.6 \\
$40 \%$ & 24.6 & 72.7 & 4.1 & 38.7 & 51.3 \\
$45 \%$ & 15.6 & 56.2 & 5.5 & 46.7 & 60.6 \\
$50 \%$ & 25.7 & 43.9 & 23.5 & 45.3 & 83.4 \\
$55 \%$ & 23.1 & 34.8 & 34.8 & 23.4 & 97.4 \\
$60 \%$ & 29.9 & 35.4 & 20.4 & 35.2 & 97.9 \\
\hline
\end{tabular}

Notes. The maximum multipole used to compute $\chi_{h}^{2}$ is $\ell_{\max }=10$.

Table 5. Probability to exceed $\chi_{h}^{2}$ for WMAP as a function of the sky fraction.

\begin{tabular}{lccccc}
\hline \hline & \multicolumn{5}{c}{ PTE [\%] } \\
\cline { 2 - 6 } Sky fraction & TE & EE & BB & TB & EB \\
\hline $30 \%$ & 64.3 & 71.7 & 50.3 & 59.9 & 92.6 \\
$35 \%$ & 91.3 & 32.5 & 81.3 & 29.0 & 97.2 \\
$40 \%$ & 84.1 & 70.8 & 79.4 & 23.2 & 90.4 \\
$45 \%$ & 82.2 & 91.0 & 92.6 & 19.5 & 61.0 \\
$50 \%$ & 74.8 & 73.6 & 84.5 & 22.8 & 36.2 \\
$55 \%$ & 81.7 & 95.3 & 65.3 & 21.3 & 50.0 \\
$60 \%$ & 70.7 & 94.2 & 58.3 & 21.1 & 83.1 \\
$65 \%$ & 57.5 & 92.2 & 66.2 & 20.4 & 75.2 \\
$70 \%$ & 53.2 & 85.8 & 69.7 & 34.9 & 78.3 \\
$75 \%$ & 55.7 & 85.4 & 67.7 & 37.5 & 63.5 \\
\hline
\end{tabular}

Notes. The maximum multipole used to compute $\chi_{h}^{2}$ is $\ell_{\max }=10$.

Table 6. Probability to exceed $\chi_{h}^{2}$ for the WMAP+LFI dataset as a function of the sky fraction.

\begin{tabular}{lccccc}
\hline \hline & \multicolumn{5}{c}{ PTE [\%] } \\
\cline { 2 - 6 } Sky fraction & TE & EE & BB & TB & EB \\
\hline $35 \%$ & 41.8 & 80.6 & 11.2 & 15.9 & 11.4 \\
$40 \%$ & 55.6 & 92.5 & 25.8 & 14.7 & 35.1 \\
$45 \%$ & 37.6 & 93.5 & 17.4 & 18.0 & 12.7 \\
$50 \%$ & 26.7 & 86.4 & 37.9 & 11.1 & 27.8 \\
$54 \%$ & 31.9 & 91.6 & 36.6 & 5.8 & 33.5 \\
$59 \%$ & 40.4 & 84.3 & 25.7 & 4.9 & 22.2 \\
$63 \%$ & 35.3 & 85.3 & 37.1 & 12.4 & 50.2 \\
$66 \%$ & 69.0 & 98.4 & 13.2 & 38.9 & 69.1 \\
$70 \%$ & 72.3 & 97.8 & 26.9 & 50.3 & 74.1 \\
$75 \%$ & 77.1 & 96.4 & 30.2 & 50.3 & 80.5 \\
\hline
\end{tabular}

Notes. The value of maximum multipole used is fixed to $\ell_{\max }=10$.

above $2.5 \sigma$ : only 3 out of a total 140 analysed multipoles. As we explain in Sect. 7, the $54 \%$ mask also represents a robust choice for the likelihood analysis. The results of the PTEs computation for the combined dataset analysed in the $54 \%$ mask are reported in Tables 7 and 8.

The spectra for WMAP, LFI and WMAP+LFI are shown in Fig. 8, in their own 50\%, 50\%, and 54\% masks, respectively.

\section{Likelihood and validation}

In this section, we show the results of additional consistency tests performed at the level of parameter estimation. This allows 
Table 7. Probability to exceed $\chi_{h}^{2}$ for the combined dataset WMAP+LFI for different choices of $\ell_{\max }$.

\begin{tabular}{lccc}
\hline \hline & \multicolumn{3}{c}{ PTE [\%] } \\
\cline { 2 - 4 } \multicolumn{1}{c}{ Spectrum } & $\ell_{\max }=10$ & $\ell_{\max }=15$ & $\ell_{\max }=29$ \\
\hline TE & 31.9 & 54.2 & 66.5 \\
EE & 91.6 & 98.4 & 98.5 \\
BB & 36.6 & 32.8 & 14.8 \\
TB & 5.8 & 12.9 & 32.7 \\
EB & 33.5 & 58.7 & 56.2 \\
\hline
\end{tabular}

Notes. Here the mask used to extract the power spectra is the combined mask with $f_{\text {sky }}=54 \%$.

Table 8. Probability to exceed $\chi_{h}^{2}$ for the WMAP+LFI dataset $\ell$-by- $\ell$.

\begin{tabular}{lccccc}
\hline \hline & \multicolumn{5}{c}{ PTE [\%] } \\
\cline { 2 - 6 } Mulitpole & TE & EE & BB & TB & EB \\
\hline 2 & 21.1 & 71.9 & 98.9 & 51.9 & 34.4 \\
3 & 20.6 & 26.8 & 11.8 & 5.7 & 7.2 \\
4 & 87.0 & 77.8 & 65.9 & 33.7 & 68.4 \\
5 & 6.0 & 56.5 & 27.2 & 17.3 & 48.3 \\
6 & 44.9 & 44.9 & 60.9 & 49.0 & 4.5 \\
7 & 99.3 & 36.4 & 94.4 & 1.7 & 90.6 \\
8 & 33.3 & 73.1 & 6.5 & 11.0 & 20.0 \\
9 & 16.4 & 36.6 & 18.9 & 40.4 & 55.0 \\
10 & 63.8 & 79.0 & 30.7 & 66.6 & 97.1 \\
11 & 20.8 & 95.4 & 91.1 & 24.7 & 98.3 \\
12 & 47.1 & 53.1 & 33.0 & 27.9 & 67.3 \\
13 & 85.4 & 53.7 & 13.8 & 38.2 & 24.7 \\
14 & 70.3 & 48.5 & 61.7 & 58.9 & 50.6 \\
15 & 73.1 & 72.9 & 19.1 & 94.5 & 94.6 \\
16 & 94.3 & 71.1 & 84.4 & 42.2 & 43.1 \\
17 & 8.0 & 21.4 & 50.1 & 28.8 & 64.0 \\
18 & 93.7 & 73.1 & 0.9 & 59.0 & 71.5 \\
19 & 60.2 & 60.5 & 55.5 & 38.4 & 26.1 \\
20 & 80.6 & 48.1 & 96.1 & 14.5 & 18.5 \\
21 & 24.0 & 38.6 & 84.3 & 43.1 & 49.1 \\
22 & 81.0 & 72.0 & 70.6 & 81.3 & 54.6 \\
23 & 19.6 & 62.0 & 0.7 & 60.7 & 1.0 \\
24 & 41.0 & 13.3 & 74.1 & 85.1 & 67.9 \\
25 & 84.0 & 33.1 & 60.8 & 71.8 & 38.4 \\
26 & 9.9 & 27.1 & 13.2 & 36.0 & 31.4 \\
27 & 66.5 & 30.1 & 93.9 & 68.8 & 47.7 \\
28 & 29.8 & 86.1 & 94.4 & 26.6 & 33.4 \\
29 & 60.3 & 38.8 & 22.5 & 12.3 & 95.1 \\
\hline & & & & & \\
& & & & &
\end{tabular}

Notes. Here the mask used to extract the power spectra is the combined mask with $f_{\text {sky }}=54 \%$.

us to test and validate both the datasets produced and the likelihood algorithm.

Parameter estimates are obtained from the likelihood function in Eq. (4). Since we are using low-resolution maps with $N_{\text {side }}=16$, only the $C_{\ell}$ 's from $\ell=2$ to $\ell_{\text {cut }}=29$ are varied in accordance to the theoretical model that is being tested, when computing the signal covariance matrix; multipoles between $\ell_{\text {cut }}+1=30$ and $\ell_{\max }=64$ are instead fixed to a fiducial $\Lambda$ CDM spectrum (Page et al. 2007; Planck Collaboration XI 2016; Planck Collaboration V 2020). We follow the procedure described in Sect. 4 in order to marginalize over the regularization noise.

As consistency test for the likelihood, we explore the stability of the reionization optical depth $\tau$ constraints with respect the mask used for cosmological parameter estimation. Thus, keeping

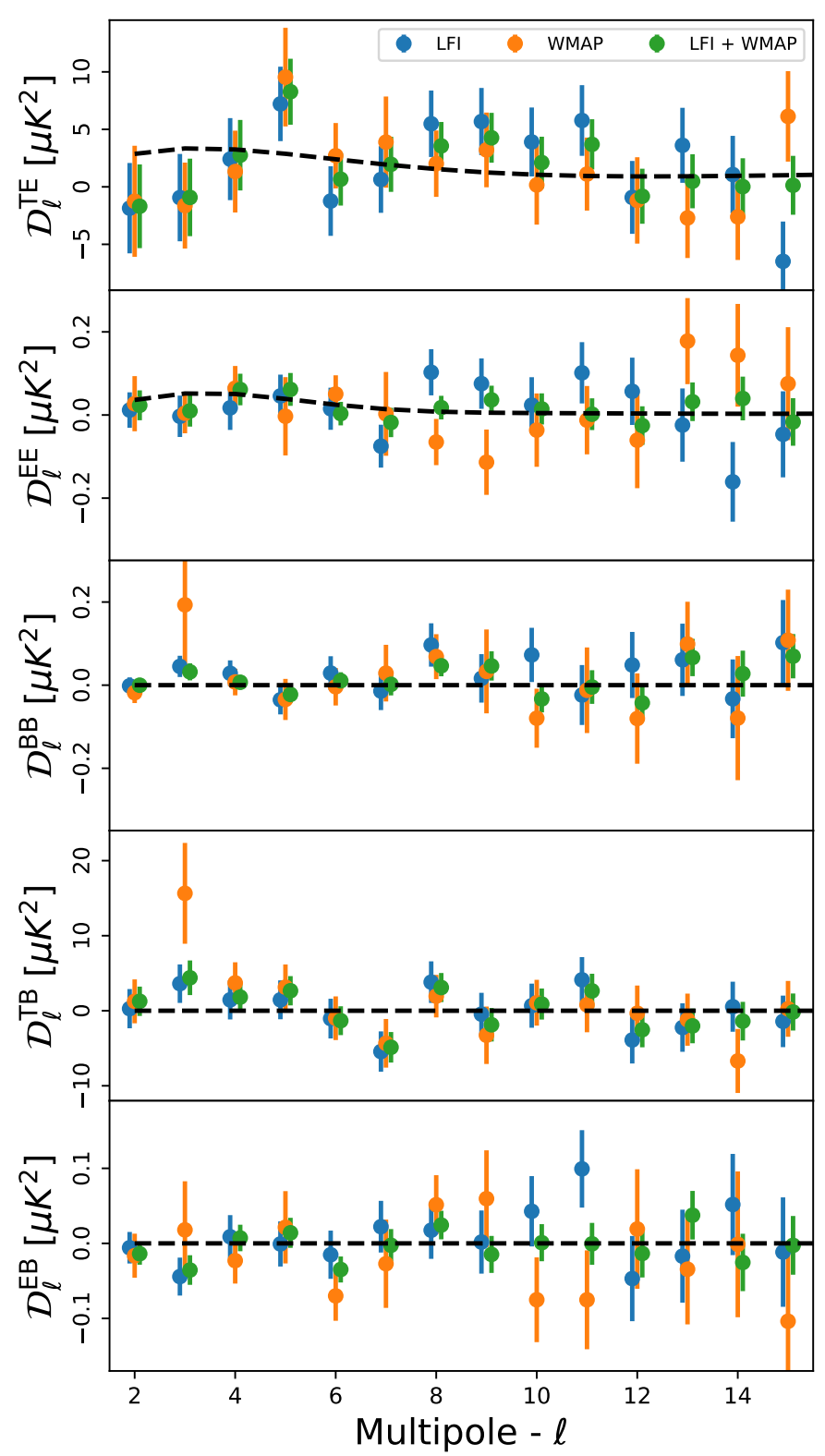

Fig. 8. Polarization power spectra of the LFI $70 \mathrm{GHz}$, WMAP bands and WMAP+LFI. The sky fractions used are respectively 50\%, 50\%, and $54 \%$. The dashed lines represent a $\Lambda \mathrm{CDM}$ power spectra corresponding to an optical depth value of $\tau=0.065$.

fixed the underlying datasets (i.e., map and associated covariance matrix) we only change the cosmological parameter mask used in Eq. (4). The results of these test are reported in Figs. 9-11, respectively, for LFI, WMAP and WMAP+LFI. Visually all the $\tau$ constraints are nicely compatible with each other for LFI and WMAP. For the WMAP+LFI, the $\tau$ posteriors are still visually compatible with each other, but we observe a clear trend towards high values of $\tau$ for large sky fractions. It is worth mentioning that all the masks we are using for a given dataset are nested, and largely overlapped, so relying on a simple visual comparison can be misleading and we need a more accurate statistical test to assess consistency. Thus, for each dataset, we generated a Monte Carlo of 1000 CMB maps, with $\tau=0.065$ and a set of realistic noise simulations extracted from the noise covariance matrix of the cleaned datasets (Eqs. (5) and (13)) through Cholesky decomposition. For every mask, we processed all those 


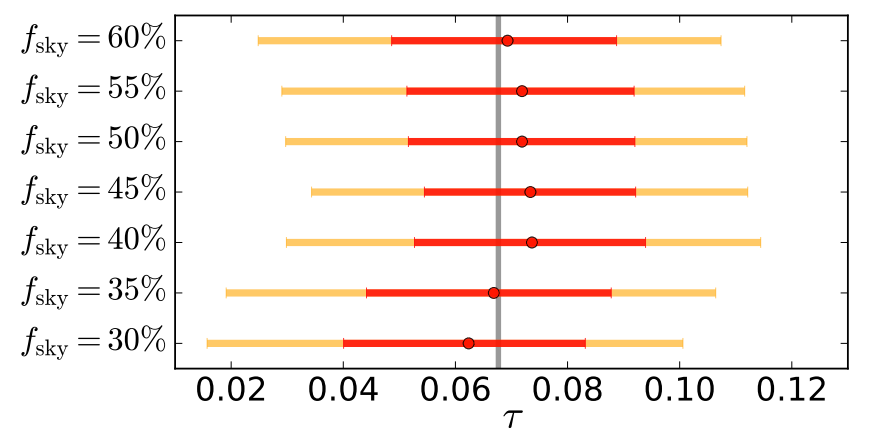

Fig. 9. Estimates of $\tau$ from the LFI $70 \mathrm{GHz}$ map analysed in different masks. In this and in the following plots, the points represent the bestfit values and the red and yellow bars represent the $68 \%$ and $95 \%$ C.L., respectively. The vertical grey line represents the best-fit value for the WMAP+LFI baseline dataset, which uses $54 \%$ of the sky; see text for details.

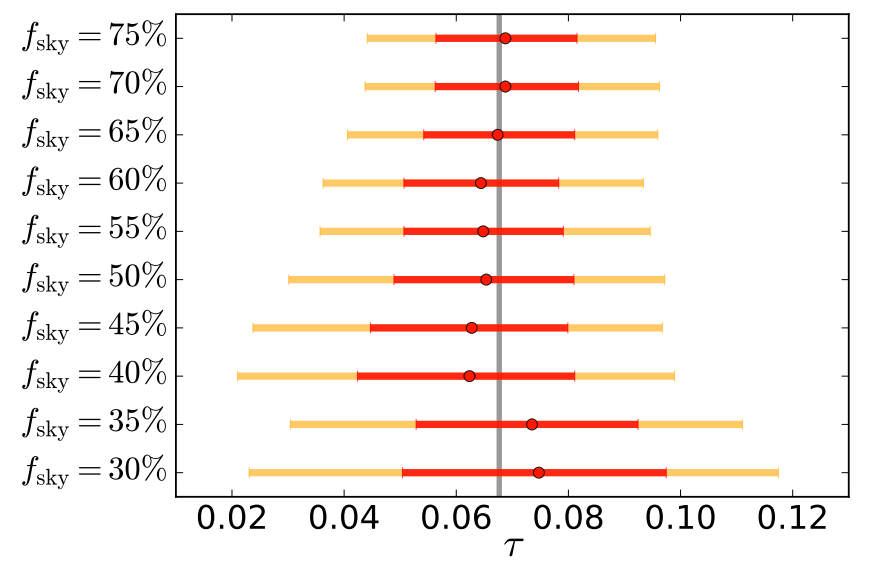

Fig. 10. Same as Fig. 9 but for the combination of WMAP $K a, Q$ and $V$ bands.

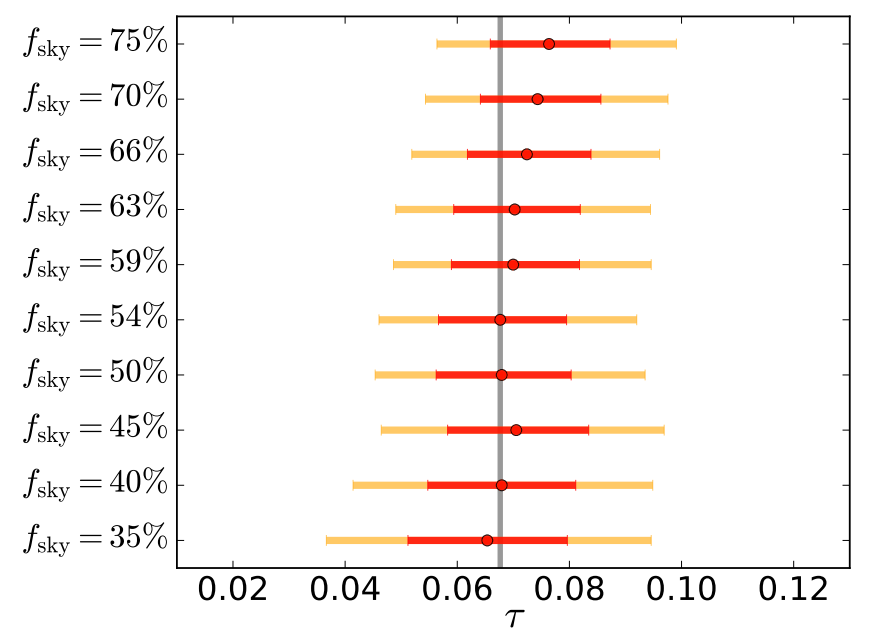

Fig. 11. Same as Fig. 9 but for the WMAP+LFI dataset.

maps through a pixel-based likelihood algorithm implementing the function in Eq. (4), fitting $\tau$ and $\ln \left(10^{10} A_{\mathrm{s}}\right)$ from a grid of models. All the other $\Lambda \mathrm{CDM}$ parameters were kept fixed to the best-fit of Pagano et al. (2020). Then we were able to build the statistics of the difference $\Delta \tau_{i j} \equiv\left|\tau_{i}-\tau_{j}\right|$ between the $\tau$ estimates for each pair of masks $\{i, j\}$, and, finally, compare this with the values of $\Delta \tau_{i j}$ obtained from the real data
In Table 9, we report the PTEs for the three datasets analyzed, defined as the percentage of simulations that have an absolute parameter shift larger than the same quantity measured on the data. As explained above, the simulations used for this test contain only the CMB signal and noise drawn by cleaned map covariance matrix. We note that foreground residuals and, thus, chance correlations between such residuals and noise realizations are not included in the simulations; this makes the test conservative since it is more difficult to pass. The scatter we see on the data is perfectly compatible with the signal plus noise simulations, independently for LFI and WMAP, and for all the sky fractions considered. The WMAP+LFI dataset, instead, shows mild failures for sky fractions larger than $60 \%$. This comes as some of a surprise since the corresponding results based on WMAP show excellent PTEs (see, e.g., Cols. 2 and 3 of Table 9). Again, we verified that the shift between the $\Delta \tau$ for WMAP and WMAP+LFI are compatible with what is seen in our signal plus noise simulations. We find that all the shifts are within $2 \sigma$. This indicates that as far as $\tau$ is concerned, all masks return consistent values and may be thus used in the analysis. However, we remark that there is a clear trend towards larger values of $\tau$ for masks with a sky fraction larger than $f_{\text {sky }}=63 \%$ (again, see Fig. 11). Based on these considerations and on the fact that this is the one performing better in the $\ell$-by- $\ell$ tests described in Sect. 6 , we opt for a conservative choice and select the $54 \%$ mask as the baseline for WMAP+LFI. This dataset provides an error on $\tau$ that is $12 \%$ smaller than the one obtained from WMAP on the $75 \%$ mask.

For the baseline mask, in Table 10, we report the constraints on $\tau, \ln \left(10^{10} A_{\mathrm{s}}\right)$, both with $r=0$ and variable $r$ from the lowmultipole dataset alone, having fixed the other $\Lambda$ CDM parameters to the best-fit of Pagano et al. (2020). In the next section, we offer a detailed discussion on the $\tau$ constraints and its consequences for the cosmological scenario.

\section{Reionization constraints}

The CMB large-scale polarization data provide an almost direct measurement of the optical depth to reionization, being $C_{\ell}^{\mathrm{EE}} \propto$ $\tau^{2}$ and $C_{\ell}^{\mathrm{TE}} \propto \tau$ for multipoles $\ell \lesssim 20$. In this section, we use the WMAP+LFI dataset in polarization, together with the Commander 2018 solution in temperature, to derive updated constraints on $\tau$ from CMB measurements at low frequencies.

For the cosmological parameter tests presented in this paper, we adopted the reionization model given in Lewis (2008). This is the default model in $\mathrm{camb}^{4}$ and it has been used for the Planck baseline cosmological results (TANH). In this model, the phase change in the intergalactic medium from the almost completely neutral state (up to a residual ionization fraction of $10^{-4}$, remaining after recombination) to the ionized state is described as a sharp transition. The hydrogen reionization is assumed to happen simultaneously with the first reionization of helium, whereas the second reionization of helium is fixed at a redshift of $z=3.5$ and is, again, described as a sharp transition. This choice is motivated by the expectations drawn from quasar spectra. Nevertheless, we expect the modeling of the helium double ionization to have a minor impact on the final results because varying the corresponding reionization redshift between 2.5 and 4.5 changes the total optical depth by less than $1 \%$ (Planck Collaboration Int. XLVII 2016). For the tests

\footnotetext{
4 https://camb.info
} 
Table 9. Consistency of the $\tau$ parameter values estimated on different masks.

\begin{tabular}{|c|c|c|c|c|c|}
\hline \multicolumn{2}{|c|}{ LFI PTE [\%] } & \multicolumn{2}{|c|}{ WMAP PTE [\%] } & \multicolumn{2}{|c|}{ WMAP+LFI PTE [\%] } \\
\hline Masks & $|\Delta \tau|$ & Masks & $|\Delta \tau|$ & Masks & $|\Delta \tau|$ \\
\hline $30 \%-35 \%$ & 49.0 & $30 \%-35 \%$ & 84.3 & $35 \%-40 \%$ & 56.9 \\
\hline $30 \%-40 \%$ & 21.3 & $30 \%-40 \%$ & 21.1 & $35 \%-45 \%$ & 38.0 \\
\hline $30 \%-45 \%$ & 27.2 & $30 \%-45 \%$ & 33.4 & $35 \%-50 \%$ & 72.3 \\
\hline $30 \%-50 \%$ & 36.4 & $30 \%-50 \%$ & 49.0 & $35 \%-54 \%$ & 76.0 \\
\hline $30 \%-55 \%$ & 40.3 & $30 \%-55 \%$ & 49.7 & $35 \%-59 \%$ & 55.9 \\
\hline $30 \%-60 \%$ & 54.0 & $30 \%-60 \%$ & 49.6 & $35 \%-63 \%$ & 54.1 \\
\hline $35 \%-40 \%$ & 21.2 & $30 \%-65 \%$ & 65.6 & $35 \%-66 \%$ & 37.6 \\
\hline $35 \%-45 \%$ & 33.4 & $30 \%-70 \%$ & 71.6 & $35 \%-70 \%$ & 26.5 \\
\hline $35 \%-50 \%$ & 52.8 & $30 \%-75 \%$ & 71.4 & $35 \%-75 \%$ & 17.6 \\
\hline $35 \%-55 \%$ & 56.6 & $35 \%-40 \%$ & 15.9 & $40 \%-45 \%$ & 46.0 \\
\hline $35 \%-60 \%$ & 79.6 & $35 \%-45 \%$ & 22.8 & $40 \%-50 \%$ & 97.5 \\
\hline $40 \%-45 \%$ & 95.8 & $35 \%-50 \%$ & 43.1 & $40 \%-54 \%$ & 96.2 \\
\hline $40 \%-50 \%$ & 75.5 & $35 \%-55 \%$ & 48.8 & $40 \%-59 \%$ & 74.9 \\
\hline $40 \%-55 \%$ & 80.2 & $35 \%-60 \%$ & 47.3 & $40 \%-63 \%$ & 71.7 \\
\hline $40 \%-60 \%$ & 55.1 & $35 \%-65 \%$ & 63.4 & $40 \%-66 \%$ & 48.5 \\
\hline $45 \%-50 \%$ & 69.7 & $35 \%-70 \%$ & 72.7 & $40 \%-70 \%$ & 32.9 \\
\hline $45 \%-55 \%$ & 76.3 & $35 \%-75 \%$ & 73.7 & $40 \%-75 \%$ & 18.6 \\
\hline $45 \%-60 \%$ & 50.6 & $40 \%-45 \%$ & 91.5 & $45 \%-50 \%$ & 34.6 \\
\hline $50 \%-55 \%$ & 95.6 & $40 \%-50 \%$ & 67.0 & $45 \%-54 \%$ & 49.6 \\
\hline $50 \%-60 \%$ & 58.3 & $40 \%-55 \%$ & 79.1 & $45 \%-59 \%$ & 88.7 \\
\hline \multirow[t]{25}{*}{$55 \%-60 \%$} & 40.8 & $40 \%-60 \%$ & 83.5 & $45 \%-63 \%$ & 96.8 \\
\hline & - & $40 \%-65 \%$ & 64.5 & $45 \%-66 \%$ & 68.7 \\
\hline & - & $40 \%-70 \%$ & 56.2 & $45 \%-70 \%$ & 46.9 \\
\hline & - & $40 \%-75 \%$ & 58.1 & $45 \%-75 \%$ & 29.7 \\
\hline & - & $45 \%-50 \%$ & 63.1 & $50 \%-54 \%$ & 92.9 \\
\hline & - & $45 \%-55 \%$ & 78.1 & $50 \%-59 \%$ & 58.5 \\
\hline & - & $45 \%-60 \%$ & 83.6 & $50 \%-63 \%$ & 56.1 \\
\hline & - & $45 \%-65 \%$ & 59.2 & $50 \%-66 \%$ & 29.1 \\
\hline & - & $45 \%-70 \%$ & 52.6 & $50 \%-70 \%$ & 16.6 \\
\hline & - & $45 \%-75 \%$ & 55.1 & $50 \%-75 \%$ & 7.7 \\
\hline & - & $50 \%-55 \%$ & 87.3 & $54 \%-59 \%$ & 32.7 \\
\hline & - & $50 \%-60 \%$ & 83.3 & $54 \%-63 \%$ & 37.8 \\
\hline & - & $50 \%-65 \%$ & 77.9 & $54 \%-66 \%$ & 13.4 \\
\hline & - & $50 \%-70 \%$ & 66.5 & $54 \%-70 \%$ & 6.5 \\
\hline & - & $50 \%-75 \%$ & 68.7 & $54 \%-75 \%$ & 3.2 \\
\hline & - & $55 \%-60 \%$ & 87.9 & $59 \%-63 \%$ & 78.6 \\
\hline & - & $55 \%-65 \%$ & 55.1 & $59 \%-66 \%$ & 18.6 \\
\hline & - & $55 \%-70 \%$ & 45.0 & $59 \%-70 \%$ & 8.0 \\
\hline & - & $55 \%-75 \%$ & 49.9 & $59 \%-75 \%$ & 3.4 \\
\hline & - & $60 \%-65 \%$ & 33.4 & $63 \%-66 \%$ & 7.2 \\
\hline & - & $60 \%-70 \%$ & 30.5 & $63 \%-70 \%$ & 3.3 \\
\hline & - & $60 \%-75 \%$ & 36.6 & $63 \%-75 \%$ & 1.5 \\
\hline & - & $65 \%-70 \%$ & 59.9 & $66 \%-70 \%$ & 14.0 \\
\hline & - & $65 \%-75 \%$ & 66.5 & $66 \%-75 \%$ & 4.1 \\
\hline & - & $70 \%-75 \%$ & 95.7 & $70 \%-75 \%$ & 12.2 \\
\hline
\end{tabular}

Notes. For each pair of masks defined in Sect. 3, we report the percentage of simulations with absolute parameter shift larger than the same quantity measured on the data. Each column corresponds to a different dataset, LFI (left), WMAP (center), and WMAP+LFI (right).

presented in this paper, we did not explore different reionization models, however, it has been shown in Planck Collaboration VI (2020) that $\tau$ constraints from the latest Planck data have little sensitivity with regard to the actual details of the reionization history. Furthermore, earlier claims by Heinrich \& $\mathrm{Hu}$ (2018) of a mild evidence, in Planck 2015 LFI data, for a more complex model of the ionization fraction, with hints of early reionization, have not been confirmed by alternate analyses of the same data set (e.g., Villanueva-Domingo et al. 2018; Hazra \& Smoot 2017; Dai et al. 2019; Hazra et al. 2020). In particular, Millea \& Bouchet (2018) have shown how the signifi-
Table 10. Constraints on $\ln \left(10^{10} A_{\mathrm{s}}\right), \tau$, and $r$ from the WMAP+LFI likelihood.

\begin{tabular}{lcc}
\hline \hline \multicolumn{1}{c}{ Parameter } & $\Lambda \mathrm{CDM}$ & $\Lambda \mathrm{CDM}+r$ \\
\hline $\ln \left(10^{10} A_{\mathrm{s}}\right)$ & $2.978 \pm 0.050$ & $2.82_{-0.08}^{+0.15}$ \\
$\tau$ & $0.069_{-0.012}^{+0.011}$ & $0.067_{-0.012}^{+0.011}$ \\
$r_{0.002}$ & $\ldots$ & $\leq 0.79$ \\
$10^{9} A_{\mathrm{s}} e^{-2 \tau}$ & $1.715_{-0.092}^{+0.081}$ & $1.48_{-0.14}^{+0.20}$ \\
\hline
\end{tabular}

Notes. We show mean and $68 \%$ confidence levels. For $r$, the $95 \%$ upper limit is shown.

cance of those findings has been likely overestimated due to the choice of unphysical priors.

Having fixed the reionization model, first of all, we want to study the constraints from the large scales alone. Using the pixel-based likelihood framework of Sect. 7 (lowTEB), we only fit for $\tau, \ln \left(10^{10} A_{\mathrm{s}}\right)$, and $r$, while keeping all the other $\Lambda \mathrm{CDM}$ parameters fixed to the best-fit values given in Pagano et al. (2020). Our results are shown in Table 10, where the parameter, $r$, is estimated at a scale of $k=0.002 \mathrm{Mpc}^{-1}$. The derived constraint on $\tau$ is

$\tau=0.069_{-0.012}^{+0.011} \quad(68 \%$, lowTEB $)$,

which corresponds to a $5.8 \sigma$ detection from the low-frequency CMB polarization data.

We then extended the analysis to include data from the small scales, specifically adding the Planck 2018 likelihood for TT, TE, EE angular power spectra (Planck Collaboration V 2020). This time, we let all the six base $\Lambda$ CDM parameters vary, and we sampled from the space of possible cosmological parameters with an MCMC exploration using CosmoMC (Lewis \& Bridle 2002). The reionization optical depth estimated in this case is ${ }^{5}$ :

$\tau=0.074_{-0.011}^{+0.010} \quad(68 \%, \mathrm{TT}, \mathrm{TE}, \mathrm{EE})$.

The parameter constraints we derived for pure $\Lambda \mathrm{CDM}$ are given in Table 11, where, for the purposes of comparison, we also report the Planck 2018 baseline results. The two compared datasets differ by the low- $\ell$ likelihoods. In one case, there is the pixel-based likelihood developed in this paper (lowTEB), while in the other case, the low- $\ell$ likelihood is a combination of the Blackwell-Rao estimator for the Commander temperature solution and the E-mode power spectrum based Planck Legacy HFI likelihood (lowE). The latter likelihood provides a constraint on $\tau$ that is about 1.5 times tighter and $1.4 \sigma$ lower in value than the one we obtain from the WMAP+LFI likelihood. Due to the well known degeneracy between $A_{s}$ and $\tau$, this also translates to a $33 \%$ tighter constraint on $\ln \left(10^{10} A_{\mathrm{s}}\right)$ and $1.8 \sigma$ lower in value. All the other cosmological parameters, rather, are in good agreement, differing by at most $36 \%$ of the $\sigma$. A similar tendency is also found when comparing Table 11 with an analogous analysis shown in Pagano et al. (2020).

Comparing the constraints from Tables 10 and 11 , we note that the values of $\ln \left(10^{10} A_{\mathrm{s}}\right)$ and $\tau$ derived from the large scales alone are $1.9 \sigma$ and $0.4 \sigma$ lower, respectively. This behaviour was first noticed in Planck Collaboration XI (2016) and it is known to be induced by the low- $\ell$ anomaly, that is, the power deficit in the measured $T T$ power spectrum with respect to the best-fit model at multipoles between $\ell=20$ and 30. When limiting the analysis to the large scales, that is, to multipoles up to 30 , the

5 In the following, the presence of the lowTEB dataset should be always understood. 
U. Natale et al.: Large scale CMB polarization likelihood from WMAP and Planck LFI legacy maps

Table 11. Parameter constraints for the $\Lambda$ CDM cosmology (as defined in Planck Collaboration XVI 2014), illustrating the impact of replacing the low- $\ell$ baseline Planck 2018 likelihood (lowE) with the WMAP+LFI likelihood presented in this paper (lowTEB).

\begin{tabular}{lcccc}
\hline \hline \multicolumn{1}{c}{ Parameter } & $\begin{array}{c}\text { TT+lowE } \\
68 \% \text { limits }\end{array}$ & $\begin{array}{c}\text { TT+lowTEB } \\
68 \% \text { limits }\end{array}$ & $\begin{array}{c}\text { TTTEEE+lowE } \\
68 \% \text { limits }\end{array}$ & $\begin{array}{c}\text { TTTEEE+lowTEB } \\
68 \% \text { limits }\end{array}$ \\
\hline$\Omega_{\mathrm{b}} h^{2}$ & $0.02212 \pm 0.00022$ & $0.02218 \pm 0.00022$ & $0.02236 \pm 0.00015$ & $0.02241 \pm 0.00015$ \\
$\Omega_{\mathrm{c}} h^{2}$ & $0.1206 \pm 0.0021$ & $0.1200 \pm 0.0021$ & $0.1202 \pm 0.0014$ & $0.1197 \pm 0.0014$ \\
$100 \theta_{\mathrm{MC}}$ & $1.04077 \pm 0.00047$ & $1.04086 \pm 0.00047$ & $1.04090 \pm 0.00031$ & $1.04096 \pm 0.00031$ \\
$\tau$ & $0.0522 \pm 0.0080$ & $0.071_{-0.011}^{+0.010}$ & $0.0544_{-0.0081}^{+0.0070}$ & $0.074_{-0.011}^{+0.010}$ \\
$\ln \left(10^{10} A_{\mathrm{s}}\right)$ & $3.040 \pm 0.016$ & $3.076 \pm 0.021$ & $3.045 \pm 0.016$ & $3.082 \pm 0.021$ \\
$n_{\mathrm{s}}$ & $0.9626 \pm 0.0057$ & $0.9645 \pm 0.0058$ & $0.9649 \pm 0.0044$ & $0.9664 \pm 0.0044$ \\
\hline$H_{0}$ & $66.88 \pm 0.92$ & $67.12 \pm 0.93$ & $67.27 \pm 0.60$ & $67.51 \pm 0.61$ \\
$\Omega_{\mathrm{m}}$ & $0.321 \pm 0.013$ & $0.317 \pm 0.014$ & $0.3166 \pm 0.0084$ & $0.3134 \pm 0.0084$ \\
$\Omega_{\Lambda}$ & $0.679 \pm 0.013$ & $0.683 \pm 0.013$ & $0.6834 \pm 0.0084$ & $0.6866 \pm 0.0084$ \\
$\sigma_{8}$ & $0.8118 \pm 0.0089$ & $0.825 \pm 0.010$ & $0.8120 \pm 0.0073$ & $0.8259 \pm 0.0091$ \\
$z_{\mathrm{re}}$ & $7.50 \pm 0.82$ & $9.3 \pm 1.0$ & $7.68 \pm 0.79$ & $9.51_{-0.97}^{+0.98}$ \\
$10^{9} A_{\mathrm{s}}$ & $2.092 \pm 0.034$ & $2.167_{-0.049}^{+0.043}$ & $2.101_{-0.034}^{+0.031}$ & $2.181_{-0.049}^{+0.043}$ \\
$10^{9} A_{\mathrm{s}} e^{-2 \tau}$ & $1.884 \pm 0.014$ & $1.882 \pm 0.014$ & $1.884 \pm 0.012$ & $1.882 \pm 0.012$ \\
$\mathrm{Age} / \mathrm{Gyr}$ & $13.830 \pm 0.037$ & $13.819 \pm 0.037$ & $13.800 \pm 0.024$ & $13.791 \pm 0.024$ \\
\hline
\end{tabular}

Notes. We also show the change when including the high- $\ell$ polarization likelihood in the analysis.

deficit has a high relative weight in the final solution, leading to a value of the overall amplitude of the spectrum that is lower than the one from the full analysis, which includes multipoles up to $\ell=2500$. Due to the aforementioned degeneracy, this also results in a lower value for $\tau$.

Since one of the main results of this paper is the $\tau$ constraint from the WMAP+LFI dataset, we want to further comment on the robustness of this result. In Fig. 12, we show the good agreement between the estimates of $\tau$ from LFI and WMAP separately. The two were derived using their own $f_{\text {sky }}=50 \%$ mask. The consistency between the two experiments is further confirmed by the null test that we performed, estimating $\tau$ from the half-difference map of the two data sets, LFI-WMAP. The posterior distribution for this case is reported in the same figure and it is compatible with noise, giving an upper limit of $\tau \leq 0.059$ at 95\% confidence level (CL).

Differently from the baseline low- $\ell$ Planck 2018 likelihood, which is based on the $T T, E E$, and $B B$ power spectra, the pixel-based likelihood used in this paper also includes the information contained in the $T E$ cross power spectrum. In order to investigate the impact of this extra information, we build a polarization-only version of the pixel-based likelihood, which contains only the $Q$ and $U$ maps and the $Q Q, Q U$, and $U U$ blocks of the covariance matrix, in an analogy of what was done in Planck Collaboration XI (2016). When we use this latter likelihood, we employ low- $\ell$ TT Commander likelihood based on the Blackwell-Rao estimator. The value of $\tau$ measured nulling the $T E$ cross correlation is

$\tau=0.062 \pm 0.012 \quad(68 \%$, Commander + lowP $)$,

which represents roughly a half- $\sigma$ downward shift with respect to the full TEB likelihood, already seen on the LFI only likelihood in Planck Collaboration XI (2016). Such behavior is also shown by WMAP which, on $50 \%$ sky, yields $\tau=0.055_{-0.017}^{+0.019}$ forcing $T E=0$ and $\tau=0.064_{-0.015}^{+0.017}$ with the full TEB likelihood.
For WMAP, the same behavior is also present on larger masks; for example on the $75 \%$ sky fraction we measure $\tau=0.065_{-0.014}^{+0.013}$ when $T E=0$ and $\tau=0.070_{-0.013}^{+0.012}$ when also TE is varied.

In all the previous cases, when TE is forced to zero, the $\tau$ posterior shifts closer to the HFI determination (Planck Collaboration V 2020; Pagano et al. 2020), which is based only on $E E$ estimates. Posteriors of the full pixel-based likelihood and the one without $T E$ for WMAP+LFI are shown in Fig. 13.

In order to verify if such behaviour is coherent with our error budget, we compare the shift in $\tau$ with a set of simulations. In Fig. 14 we show the histogram of $\Delta \tau$, defined as the difference between the $\tau$ estimated form the full TEB likelihood ("Full") and the $\tau$ estimated forcing $T E=0$ ("noTE"), for a Montecarlo of 1000 signal and noise simulations. This analysis shows that nullifying $T E$ still provides an unbiased estimation of $\tau$ and also that the shift observed in data is not anomalous, representing a $1.7 \sigma$ fluctuation. We also show in Fig. 15, a similar plot for the ratio of $1 \sigma$ errors defined as $\sigma_{\tau_{\text {noTE }}} / \sigma_{\tau_{\text {Full }}}$; also, in this case, the value measured on data is compatible with the simulations. This test also suggests that for this dataset, removing TE degrades $\sigma_{\tau}$ by about $5 \%$ on average.

Adding to the CMB temperature and polarization data the Planck lensing likelihood (Planck Collaboration VIII 2020) and baryon acoustic oscillation (BAO) measurements (Alam et al. 2017; Beutler et al. 2011; Ross et al. 2015) breaks the degeneracy more efficiently with the amplitude of the scalar perturbations providing

$\tau=0.0714_{-0.0096}^{+0.0087} \quad(68 \%, \mathrm{TT}, \mathrm{TE}, \mathrm{EE}+$ Lensing $+\mathrm{BAO})$.

Assuming the TANH model for the ionzation fraction the $\tau$ constrain can be directly converted into a mid-point reionization redshift of

$z_{\mathrm{re}}=9.28 \pm 0.84 \quad(68 \%, \mathrm{TT}, \mathrm{TE}, \mathrm{EE}+$ Lensing $+\mathrm{BAO})$. 


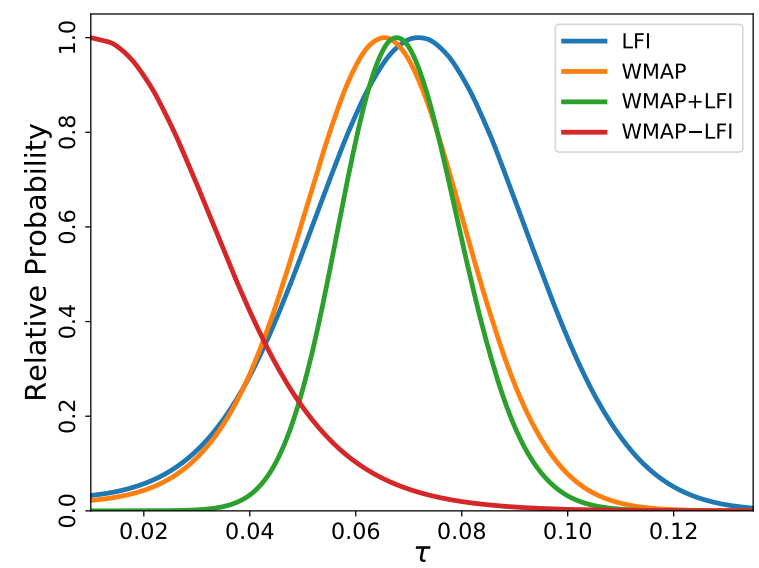

Fig. 12. Posterior distributions of $\tau$. LFI (blue) and WMAP (orange) are computed on their own 50\% mask, WMAP+LFI (green) in the union of the two, retaining $54 \%$ of the sky, while WMAP-LFI (red) is computed in their intersection, retaining $46 \%$ of the sky.

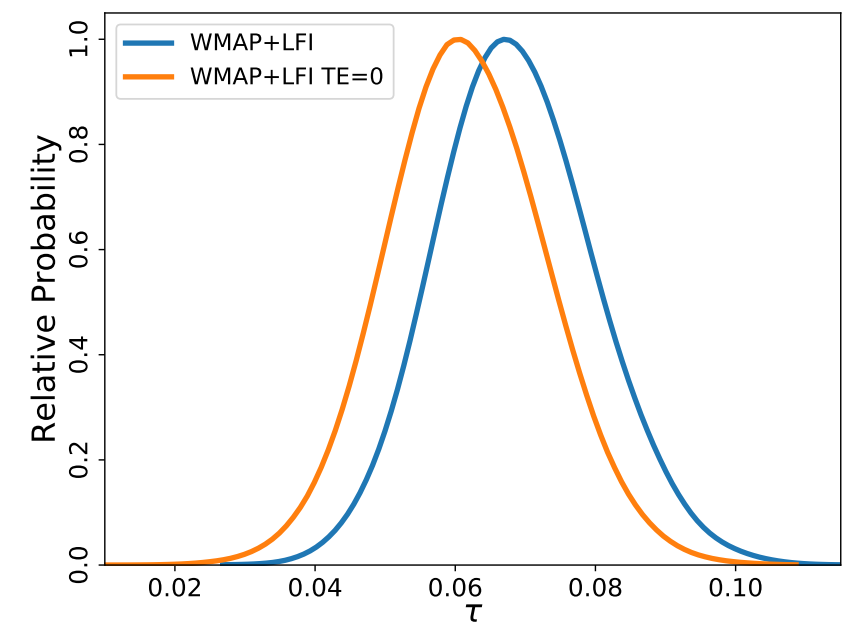

Fig. 13. Comparison of $\tau$ posterior distributions for the WMAP+LFI using the full TQU likelihood (blue) and imposing $T E=0$ in order to factorize the T and QU parts of the likelihood (orange).

This value is higher but still compatible with analogous estimates that instead use the Planck HFI based large-scale polarization likelihood, $z_{\mathrm{re}}=7.82 \pm 0.71$ (Planck Collaboration VI 2020) and $z_{\text {re }}=8.21 \pm 0.58$ (Pagano et al. 2020).

The WMAP+LFI CMB map and the corresponding covariance matrix are packaged in low- $\ell$ likelihood modules compatible with the clik infrastructure (Planck Collaboration XV 2014; Planck Collaboration ES 2013, 2015, 2018) which are made publicly available ${ }^{6}$. We provide both a likelihood module that implements Eq. (4) inverting the full covariance matrix and one that implements the Sherman-Morrison-Woodbury (SMW) formula (Golub \& Van Loan 1996) which allows us to speed up the computation by an order of magnitude (see Planck Collaboration XI 2016, Appendix B.1 for details) but does not include TB an EB. In both cases, in order to keep full compatibility with the codes of clik framework, we do not treat the regularization noise as described in Sect. 4, but instead we sum a single realization. Such noise realization has been chosen in order to have a deviation for $\ln \left(10^{10} A_{\mathrm{s}}\right)$ and $\tau$ with respect to the baseline case smaller than $1 \%$ in units of $\sigma$.

6 The WMAP+LFI likelihood module is available on https://web. fe.infn.it/ pagano/low_ell_datasets/wmap_lfi_legacy

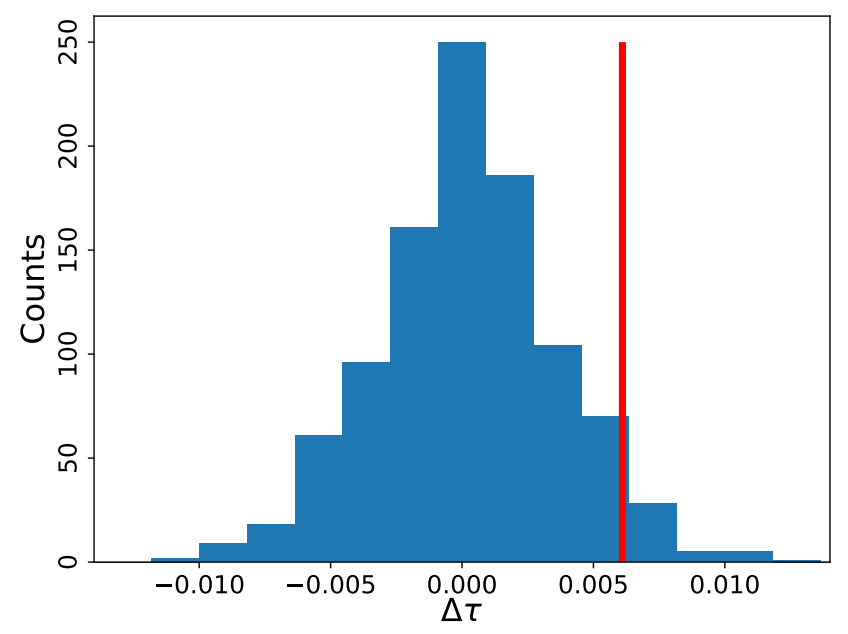

Fig. 14. Histogram of $\Delta \tau \equiv \tau_{\text {Full }}-\tau_{\text {noTE }}$ obtained analyzing a Montecarlo of 1000 simulations. The red vertical bar shows the same quantity evaluated on the data.

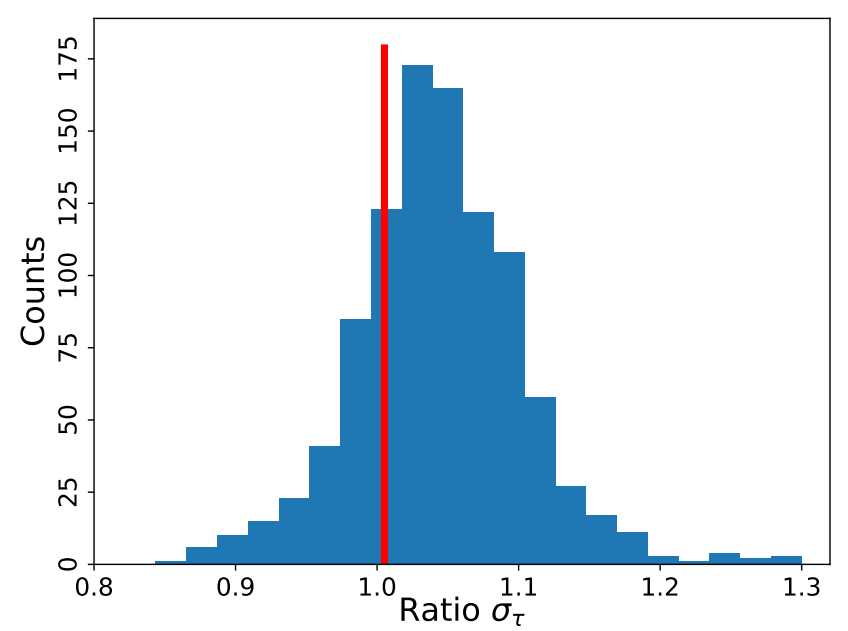

Fig. 15. Histogram of $\sigma_{\tau_{\text {noTE }}} / \sigma_{\tau_{\text {Full }}}$ obtained analyzing a Montecarlo of 1000 simulations. The red vertical bar shows the same quantity evaluated on the data.

\section{Conclusions}

In this work, we present a novel CMB pixel-space likelihood focussed on polarization at large angular scales, whose main cosmological target is the optical depth to reionization, $\tau$. The underlying dataset combines foreground-mitigated WMAP $\mathrm{Ka}$, $Q$, and $V$ bands with Planck LFI $70 \mathrm{GHz}$ channel in an optimally weighted CMB map. In the foreground cleaning of WMAP bands, we adopt the Planck $353 \mathrm{GHz}$ channel as a dust template, instead of the WMAP dust model based on starlight-derived polarization directions. As a synchrotron template, the $K$ band is used for WMAP channels, while Planck $30 \mathrm{GHz}$ is used for the $70 \mathrm{GHz}$ map. The corresponding covariance matrix is computed coherently and fed, together with the cleaned CMB map, into a pixel space likelihood, made publicly available with this paper. We produced a set of masks with increasing sky fraction and used them to test the performance of the component separation, the quality of polarization power spectra, and the overall stability of $\tau$ constraints, showing a remarkable stability among sky fractions.

For the baseline dataset, which retains $54 \%$ of the sky, the $\ell$-by- $\ell$ probability to exceed the $\chi^{2}$ of the measured angular 


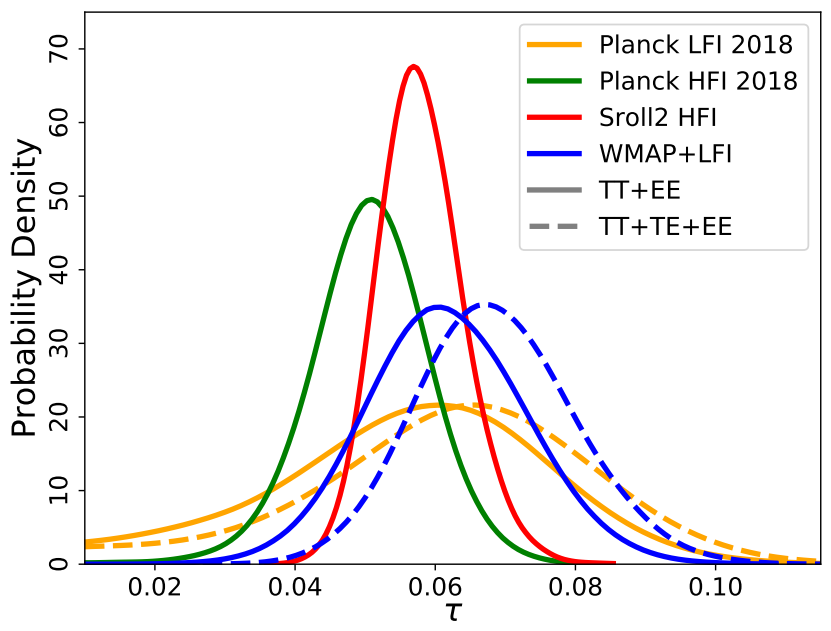

Fig. 16. Posterior distributions for $\tau$ for various datasets. Solid lines represent $\tau$ constraints ignoring $T E$, dashed lines assume full TEB likelihood. Only $\tau$ and $\ln \left(10^{10} A_{\mathrm{s}}\right)$ are sampled, whereas the remaining $\Lambda \mathrm{CDM}$ parameters are fixed to common fiducial values. The green and yellow lines show results obtained with the official Planck Legacy low$\ell$ likelihoods (Planck Collaboration V 2020). The red line is obtained running the SRoll2 likelihood (Pagano et al. 2020). The blue lines represent the results of this paper.

power spectra (PTE) does not show any major outlier, with only $\ell=18$ and 23 of BB and $\ell=23$ of EB spectra at more than $2.5 \sigma$. Consequently, the integrated PTEs are perfectly consistent with simulations both on the reionization peak only (i.e., $\ell=2 \div 10$ ) and on the full multipole range (i.e., $\ell=2 \div 29$ ).

Regarding the reionization optical depth estimation, we compared the variation of $\tau$ estimated on different sky fractions with a Montecarlo of signal plus noise, finding no significant deviations for the baseline dataset compared with other sky fractions, up to $f_{\text {sky }} \sim 70 \%$.

Sampling the parameter space with our low- $\ell$ likelihood only, we find $\tau=0.069_{-0.011}^{+0.012}$. When CMB small scales, BAO observations, and Planck lensing likelihood are included, we shrink optical depth constraint down to $\tau=$ $0.0714_{-0.0096}^{+0.0087}$. Such bounds are slightly less constraining when compared with the existing Planck HFI based likelihood (see, e.g., Planck Collaboration V 2020; Pagano et al. 2020, and our Fig. 16), yet they represent a novel measurement obtained with an independent pipeline that adopts different data and likelihood approximation and includes TE correlation, while the Planck HFI estimates are currently restricted to EE information. The $\tau$ estimates obtained with the likelihood package discussed in this paper is, in general, very compatible with the Planck HFI based constraints, with a preference for slightly higher $\tau$ values, probably driven by the inclusion of TE. This bound is also in perfect agreement with the Planck LFI Legacy likelihood (Planck Collaboration V 2020). Within the $\Lambda$ CDM model, $\tau$ can be also constrained without the use of polarization data to break the degeneracy between $A_{s}$ and $\tau$, combining temperature and weak lensing data. In this kind of analysis, the Planck collaboration found $\tau=0.080 \pm 0.025$ from temperature and lensing data and $\tau=0.078 \pm 0.016$ when BAO is added (Planck Collaboration ES 2018). Those values, despite being slightly higher than previous findings (Weiland et al. 2018; Planck Collaboration XIII 2016), are still in agreement with our constraints.

The likelihood package we provide is built on a real-space estimator and does not assume rotational invariance, but, rather, only the Gaussianity of the fields, which secures several advantages. For instance, it can also be easily used for constraining non-rotationally invariant cosmologies, including, naturally, the $\mathrm{TB}$ and EB spectra in the parameter exploration.

Acknowledgements. We acknowledge the use of camb, HEALPix and Healpy software packages, and the use of computing facilities at CINECA. We are grateful to $\mathrm{M}$. Gerbino for useful suggestions. MM is supported by the program for young researchers "Rita Levi Montalcini" year 2015. We acknowledge support from the COSMOS network (wWw . cosmosnet. it) through the ASI (Italian Space Agency) Grants 2016-24-H.0 and 2016-24-H.1-2018.

\section{References}

Alam, S., Ata, M., Bailey, S., et al. 2017, MNRAS, 470, 2617

Benabed, K., Cardoso, J. F., Prunet, S., \& Hivon, E. 2009, MNRAS, 400, 219

Bennett, C. L., Larson, D., Weiland, J. L., et al. 2013, ApJS, 208, 20

Beutler, F., Blake, C., Colless, M., et al. 2011, MNRAS, 416, 3017

Dai, W.-M., Ma, Y.-Z., Guo, Z.-K., \& Cai, R.-G. 2019, Phys. Rev. D, 99, 043524

Delouis, J. M., Pagano, L., Mottet, S., Puget, J. L., \& Vibert, L. 2019, A\&A, 629, A38

Gerbino, M., Lattanzi, M., Migliaccio, M., et al. 2020, Front. Phys., 8, 15

Golub, G. H., \& Van Loan, C. F. 1996, Matrix Computations, 3rd edn. (The Johns Hopkins University Press)

Górski, K. M., Hivon, E., Banday, A. J., et al. 2005, ApJ, 622, 759

Hazra, D. K., \& Smoot, G. F. 2017, JCAP, 1711, 028

Hazra, D. K., Paoletti, D., Finelli, F., \& Smoot, G. F. 2020, Phys. Rev. Lett., 125, 071301

Heinrich, C., \& Hu, W. 2018, Phys. Rev. D, 98, 063514

Hinshaw, G., Larson, D., Komatsu, E., et al. 2013, ApJS, 208, 19

Lattanzi, M., Burigana, C., Gerbino, M., et al. 2017, JCAP, 1702, 041

Lewis, A. 2008, Phys. Rev. D, 78, 023002

Lewis, A., \& Bridle, S. 2002, Phys. Rev. D, 66, 103511

Millea, M., \& Bouchet, F. 2018, A\&A, 617, A96

Pagano, L., Delouis, J.-M., Mottet, S., Puget, J.-L., \& Vibert, L. 2020, A\&A, 635, A99

Page, L., Hinshaw, G., Komatsu, E., et al. 2007, ApJS, 170, 335

Planck Collaboration ES 2013, The Explanatory Supplement to the Planck 2013 Results (ESA), https://www. cosmos.esa.int/web/planck/pla

Planck Collaboration ES 2015, The Explanatory Supplement to the Panck 2015 Results (ESA)

Planck Collaboration ES 2018, The Legacy Explanatory Supplement (ESA), https://www. cosmos.esa.int/web/planck/pla

Planck Collaboration XV. 2014, A\&A, 571, A15

Planck Collaboration XVI. 2014, A\&A, 571, A16

Planck Collaboration XXIII. 2014, A\&A, 571, A23

Planck Collaboration XI. 2016, A\&A, 594, A11

Planck Collaboration XIII. 2016, A\&A, 594, A13

Planck Collaboration XVI. 2016, A\&A, 594, A16

Planck Collaboration I. 2020, A\&A, 641, A1

Planck Collaboration II. 2020, A\&A, 641, A2

Planck Collaboration III. 2020, A\&A, 641, A3

Planck Collaboration IV. 2020, A\&A, 641, A4

Planck Collaboration V. 2020, A\&A, 641, A5

Planck Collaboration VI. 2020, A\&A, 641, A6

Planck Collaboration VIII. 2020, A\&A, 641, A8

Planck Collaboration Int. XLVII. 2016, A\&A, 596, A108

Ross, A. J., Samushia, L., Howlett, C., et al. 2015, MNRAS, 449, 835

Tegmark, M. 1996, MNRAS, 280, 299

Tegmark, M., \& de Oliveira-Costa, A. 2001, Phys. Rev. D, 64, 063001

Villanueva-Domingo, P., Gariazzo, S., Gnedin, N. Y., \& Mena, O. 2018, JCAP, 1804, 024

Weiland, J., Osumi, K., Addison, G., et al. 2018, ApJ, 863, 161 